\title{
Effects of brain-derived neurotrophic factor and noise on transitions in temporal lobe epilepsy in a hippocampal network
}

Liyuan Zhang, Denggui Fan, Qingyun Wang, and Gerold Baier

Citation: Chaos 28, 106322 (2018); doi: 10.1063/1.5036690

View online: https://doi.org/10.1063/1.5036690

View Table of Contents: http://aip.scitation.org/toc/cha/28/10

Published by the American Institute of Physics

\section{Articles you may be interested in}

Attractor dynamics of a Boolean model of a brain circuit controlled by multiple parameters

Chaos: An Interdisciplinary Journal of Nonlinear Science 28, 106318 (2018); 10.1063/1.5042312

Chaos versus noise as drivers of multistability in neural networks

Chaos: An Interdisciplinary Journal of Nonlinear Science 28, 106321 (2018); 10.1063/1.5043447

Modeling effects of neural fluctuations and inter-scale interactions

Chaos: An Interdisciplinary Journal of Nonlinear Science 28, 106319 (2018); 10.1063/1.5044510

Astrocyte calcium signaling: Interplay between structural and dynamical patterns

Chaos: An Interdisciplinary Journal of Nonlinear Science 28, 106320 (2018); 10.1063/1.5037153

Spiking patterns and synchronization of thalamic neurons along the sleep-wake cycle

Chaos: An Interdisciplinary Journal of Nonlinear Science 28, 106314 (2018); 10.1063/1.5039754

Bottom-up approach to torus bifurcation in neuron models

Chaos: An Interdisciplinary Journal of Nonlinear Science 28, 106317 (2018); 10.1063/1.5042078

\section{Don't let your writing keep you from getting published!}




\title{
Effects of brain-derived neurotrophic factor and noise on transitions in temporal lobe epilepsy in a hippocampal network
}

\author{
Liyuan Zhang, ${ }^{1}$ Denggui Fan, ${ }^{2}$ Qingyun Wang, ${ }^{1, a)}$ and Gerold Baier ${ }^{3}$ \\ ${ }^{1}$ Department of Dynamics and Control, Beihang University, 100191 Beijing, China \\ ${ }^{2}$ School of Mathematics and Physics, University of Science and Technology Beijing, 100083 Beijing, China \\ ${ }^{3}$ Cell and Developmental Biology, University College London, London WC1E 6BT, United Kingdom
}

(Received 18 April 2018; accepted 19 September 2018; published online 18 October 2018)

\begin{abstract}
Brain-derived neurotrophic factor (BDNF) has recently been implicated in the modulation of receptor activation leading to dynamic state transitions in temporal lobe epilepsy (TLE). In addition, the crucial role of neuronal noise in these transitions has been studied in electrophysiological experiments. However, the precise role of these factors during seizure generation in TLE is not known. Building on a previously proposed model of an epileptogenic hippocampal network, we included the actions of BDNF-regulated receptors and intrinsic noise. We found that the effects of both BDNF and noise can increase the activation of $\mathrm{N}$-methyl-D-aspartate receptors leading to excessive $\mathrm{Ca}^{2+}$ flux, which induces abnormal fast spiking and bursting. Our results indicate that the combined effects have a strong influence on the seizure-generating network, resulting in higher firing frequency and amplitude. As correlations between firing increase, the synchronization of the entire network increases, a marker of the ictogenic transitions from normal to seizures-like dynamics. Our work on the effects of BDNF dynamics in a noisy environment might lead to an improved model-based understanding of the pathological mechanisms in TLE. Published by AIP Publishing. https://doi.org/10.1063/1. 5036690
\end{abstract}

Electrophysiological data have demonstrated the capability of brain-derived neurotrophic factor (BDNF) to lead to seizure discharges in temporal lobe epilepsy (TLE). In addition, experimental evidence indicates that neuronal noise can induce the transition to seizures in TLE. Based on these findings, we modify an existing hippocampal network model by including BDNF-modulated receptors and intrinsic noise to investigate transitions to seizure dynamics. Results reveal that N-methyl-D-aspartate (NMDA) receptors are activated excessively causing maximal permeability to $\mathrm{Ca}^{2+}$, when the concentration of BDNF and the strength of noise are enhanced. These physiological processes are therefore able to induce abnormal irregular fast spiking and bursting events. Moreover, the combined effects of BDNF and noise lead to an increase of the degree of synchronization of the hippocampal network, with increased amplitudes of the membrane potentials. These computational results highlight the possible mechanisms of seizure-like transitions occurring within the hippocampus.

\section{INTRODUCTION}

Temporal lobe epilepsy (TLE) is the most prevalent refractory epilepsy characterized by complex pathogenesis. ${ }^{1}$ The dynamics within the hippocampus, which is a part of the limbic system, are considered to play a significant role in the initiation and propagation of TLE. ${ }^{2,3}$ One of the wellstudied molecules in the context of TLE is the brain-derived neurotrophic factor (BDNF) which is a neurotrophin. ${ }^{4,5}$ Clinical evidence suggests that BDNF is involved in synaptic

a)Email: nmqingyun@163.com plasticity, playing a role of a critical mediator in both learning and memory. ${ }^{6,7}$ It is reported that BDNF effectively modulates excitatory and inhibitory synaptic transmission. For example, BDNF enhances excitatory firing in the hippocampus leading to increased excitatory postsynaptic potentials (EPSPs). ${ }^{8,9}$ Previous research also investigated the impact of BDNF on neural activity within the hippocampal region called Cornu Ammonis 3 (CA3). Granule cells employ mossy fibers, which contain a high level of BDNF, to innervate pyramidal cells of CA3. ${ }^{10,11}$ This phenomenon shows the ability of BDNF to increase the excitability of principal cells. At the initial stage of TLE seizures, the level of BDNF increases and thereby enhances the activation of the BNDF's receptor TrkB. Moreover, intrahippocampal overexpression of BDNF or TrkB increases seizure susceptibility and severity. ${ }^{12,13}$

At a molecular level, the $\alpha$-amino-3-hydroxy-5-methyl4-isoxazolepropinonic acid (AMPA) receptor and the $\mathrm{N}$ methyl-D-aspartate (NMDA) receptor are the two main types of glutamate receptors. The AMPA receptor is responsible for fast excitatory transmissions, while NMDA receptor activation leads to slower post-synaptic excitation. Some key findings also indicated that BDNF-induced NMDA receptor activation could facilitate AMPA receptor responses. ${ }^{14,15}$ During the process of membrane depolarization, $\mathrm{Mg}^{2+}$ is expelled from the pore, allowing $\mathrm{Na}^{+}, \mathrm{K}^{+}$, and $\mathrm{Ca}^{2+}$ to pass. ${ }^{16}$ The current view is that NMDA receptor activation presents the main mechanism mediating glutamate-induced neuronal injury and ictogenesis (seizure generation). Furthermore, the presence of NMDA receptors is thought to allow maximal permeability of $\mathrm{Ca}^{2+} .{ }^{17}$ Following activation, a high amount of $\mathrm{Ca}^{2+}$ enters the neuronal cell, which could trigger a dependent pathway to induce neuronal death and thereby further worsen epilepsy-related pathology. ${ }^{18-20}$ 
Another widely studied factor that affects ictogenesis is neuronal noise. ${ }^{21-23}$ Noise is ubiquitous in both natural and engineering systems. The constructive effects of noise on signal transmission among neurons and in neural networks have been extensively studied in electrophysiological experiments and computational modeling, respectively. ${ }^{24,25}$ On the level of macroscopic brain networks, afferent noise is used to represent input from distal brain regions or non-modeled parts of the brain. ${ }^{26}$ Individual neurons can exhibit different firing patterns depending on the type of external input. ${ }^{27}$ Within neurons, conduction processes, membrane fluctuations, synaptic background activity, and stochastic gating of ion channels make the environment noisy. Sun et al. ${ }^{28}$ employed HodgkinHuxley $(\mathrm{HH})$ neurons to investigate the effects of channel noise on firing coherence in Watts-Strogatz small-world networks and found that firing coherence of a neuronal network depends on the source of channel noise. In addition, they also studied the effects of noise correlations on the mean firing rate. ${ }^{29}$ It is found that temporal correlations are beneficial for the generation of epileptiform rhythmic waves. Noise-induced alterations of neurons impact neuronal information processing and synchronization in a variety of neurological disorders, such as epilepsy and Parkinson's disease. ${ }^{30,31}$ In animal models using hippocampal slice preparations, the introduction of Gaussian noise can produce seizure-like events. ${ }^{24}$ In a mathematical model of epileptic dynamics, it was observed that the stochastic version of the model can reproduce kindled seizure dynamics even if it operates in the non-epileptic regime of the noise-free model. ${ }^{32}$ Fan et al.$^{33}$ proposed a modified, spatially-extended Taylor-Baier neural field model and identified paths between absence and tonic-clonic seizures. Dynamic analysis revealed that the level of noise qualitatively mediates the collective behavior, including abnormally synchronized oscillations of the network. ${ }^{34-37}$ In contrast, the noise level can change single-spike activities, e.g., from tonic firing to bursting discharges. ${ }^{38-44}$

Hence, attention should be paid to the effects of BDNF and noise on the transitions to seizure dynamics in the hippocampus. Here, we study these effects in a hippocampal network which includes CA3 and the adjacent dentate gyrus (DG). We impose a BDNF concentration pulse and Gaussian white noise on the DG-CA3 network and investigate the influence of the concentration strength and noise intensity. Moreover, we study the effects at the level of individual neurons and of the whole network.

We describe the network's structure in Sec. II. Then, we investigate the responses of individual neurons and of the entire network to the combined effects of BDNF and noise, respectively. Finally, some conclusions are drawn in Sec. IV.

\section{METHODS}

\section{A. Description of the modified DG-CA3 framework}

In this paper, we study a modified version of our previously proposed DG-CA3 network model. ${ }^{45,46}$ Specifically, we introduce the BDNF-regulated activation of NMDA receptors ${ }^{15}$ and $\mathrm{Ca}^{2+}$ (whose permeability is regulated by NMDA receptors)-regulated activation of AMPA receptors. ${ }^{47}$ As seen in Fig. 1, the DG region is composed of three type

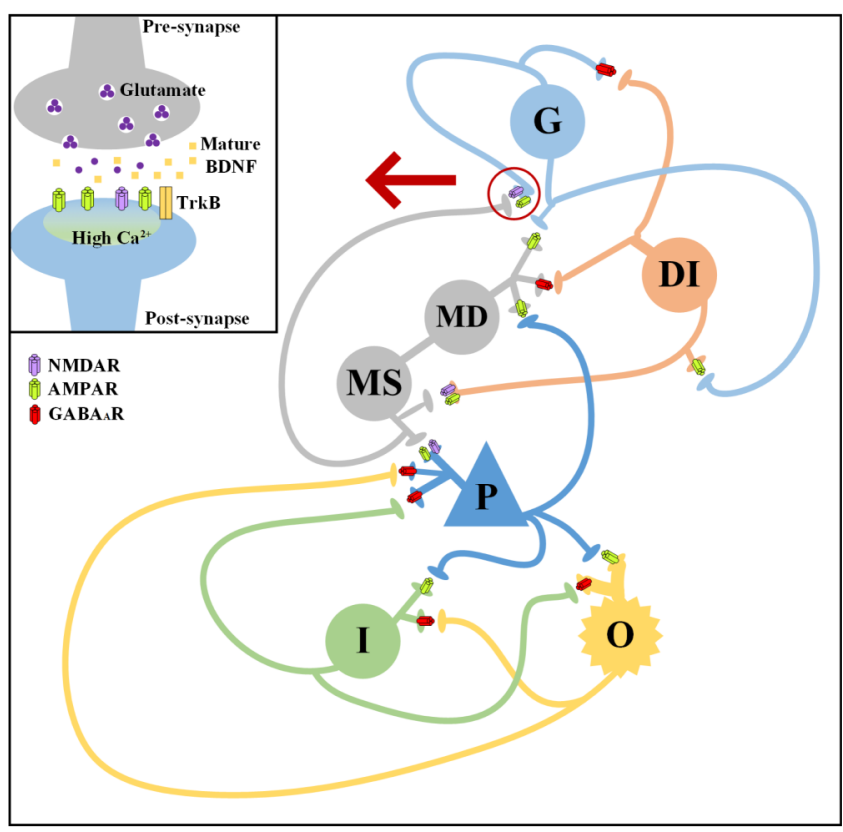

FIG. 1. Framework of the modified DG-CA3 network. The postsynaptic granule cell (G) contains NMDA type, AMPA type, and GABA type receptors. The pyramidal cell $(\mathrm{P})$ also has these three types of receptors. Like the interneuron of CA3 (I), the O-LM cell (O) and the dendrites of mossy cell (MD) lack the NMDA type receptors. In addition, DI does not contain type $\mathrm{GABA}_{\mathrm{A}}$ receptors. The zoom in the upper left corner shows synapses between $\mathrm{G}$ and the soma of mossy cell (MS) illustrating BDNF-induced synaptic activity. Glutamate is shown as dark purple spheres. Mature BDNF are shown as yellow spheres. TrkB is symbolized by yellow columns. NMDA type receptors are displayed as light purple columns, AMPA type receptors as green columns, and $\mathrm{GABA}_{\mathrm{A}}$ type receptors as red columns.

of neurons. A HH type model simulates inhibitory interneurons (DI) ${ }^{48}$ and granule cells (G) ${ }^{49}$ Representations of mossy cells (soma-MS and dendrite-MD) are constructed by a twocompartment model simplified by Pinsky and Rinzel from Trub's 19-compartment model. ${ }^{50}$ In the DG, an MS neuron interacts with $G$ and DI cells by AMPA type synapses and NMDA type synapses, respectively. G affects other neurons depending on AMPA type synapses. DI uses GABAergic type synapses to influence other neurons. In addition, CA3 is equipped with $\mathrm{HH}$-type pyramidal cells $(\mathrm{P}),{ }^{51}$ inhibitory interneurons (I), ${ }^{48}$ and O-LM cells $(\mathrm{O}) .{ }^{52}$ In this region, $\mathrm{P}$ contacts with neurons by means of AMPA type synapses. $\mathrm{O}$ and $\mathrm{I}$ connect with neurons using GABAergic synapses. ${ }^{53}$ Note that the DG region and CA3 interact with each other. In particular, DG delivers excitatory signals to CA3 through a fiber path, while the backprojection from CA3 to DG is realized with a path from $\mathrm{P}$ to $\mathrm{MD}$.

The detailed activity of the presynaptic MS neuron leading to $\mathrm{Ca}^{2+}$ influx into the postsynaptic $\mathrm{G}$ neuron through NMDA receptors is demonstrated in Fig. 1. Glutamate is released from the pre-synaptic neuron when both NMDA and AMPA receptors are activated. Mature BDNF activates TrkB, and then increases the protein production of NMDA and AMPA receptors. A strong BDNF signal enhances the activation of NMDA receptors, which leads to elevated intracellular $\mathrm{Ca}^{2+}$ concentration and increased postsynaptic currents. The detailed equations of the modified DG-CA3 network are shown in Appendixes A-G. 


\section{B. BDNF concentration pulse and noise distribution}

The BDNF protein is encoded by the BDNF gene, and its transcription is controlled by eight different promoters. ${ }^{54}$ The resultant BDNF is released from the post-synaptic membrane in an activity-dependent manner. BDNF secreted into the synaptic cleft will affect both the presynaptic and the postsynaptic neuron. In our work, we simulate BDNF from external sources in the synaptic cleft. Intracellular BDNF is not considered explicitly. The detailed BDNF concentration pulse is described as follows:

$$
\begin{aligned}
C_{B D N F}= & c_{B D N F} H\left[\sin \left(2 \pi t / \rho_{B D N F}\right)\right] \\
& \cdot\left(1-H\left\{\sin \left[2 \pi\left(t+\delta_{B D N F}\right) / \rho_{B D N F}\right]\right\}\right),
\end{aligned}
$$

where the pulse period $\rho_{B D N F}=50 \mathrm{~ms}$, the positive current $\delta_{B D N F}=5 \mathrm{~ms}$, and the pulse amplitude $c_{B D N F}$ is within the range $4-32 \mu \mathrm{mol}$, whose variation interval is $4 \mu \mathrm{mol}$ in each trial. $H(t)$ is the Heaviside function, i.e., $H(t)=1$ if $t>0$, while $H(t)=0$ if $t \leq 0$. In addition, we impose Gaussian white noise to simulate a noisy neuronal environment. The detailed expression of the noise distribution can be written as $N \sim N\left(\right.$ Mean $\left.I, \sigma^{2}\right)$, where Mean $I \in\left(2 \mu \mathrm{A} / \mathrm{cm}^{2}, 8 \mu \mathrm{A} / \mathrm{cm}^{2}\right)$ is the mean value with variation interval of $2 \mu \mathrm{A} / \mathrm{cm}^{2}$. In addition, $\sigma$ represents the standard deviation. On the one hand, the additive noise term accounts for biological phenomena like noise-induced bursts of individual neurons. On the other hand, the level of noise in the system is able to trigger its behavior, producing, for example, synchronized oscillations of the whole network, desynchronization of oscillating regimes, and it can stabilize or destabilize stationary solutions. ${ }^{55}$ Hence, these complex and biologically relevant phenomena can be studied by introducing noise terms to the model.
(A1)

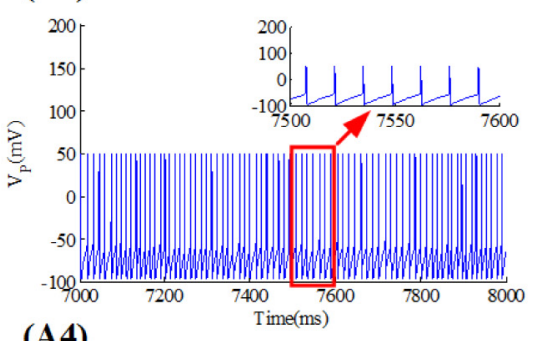

(A4)

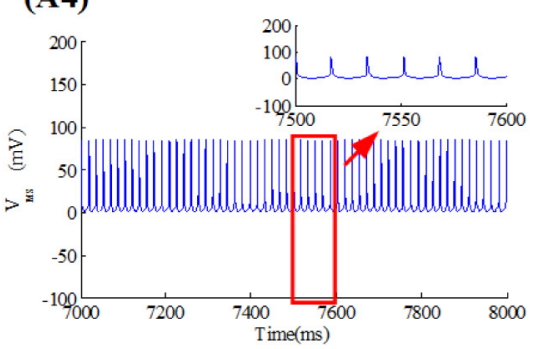

(B1)
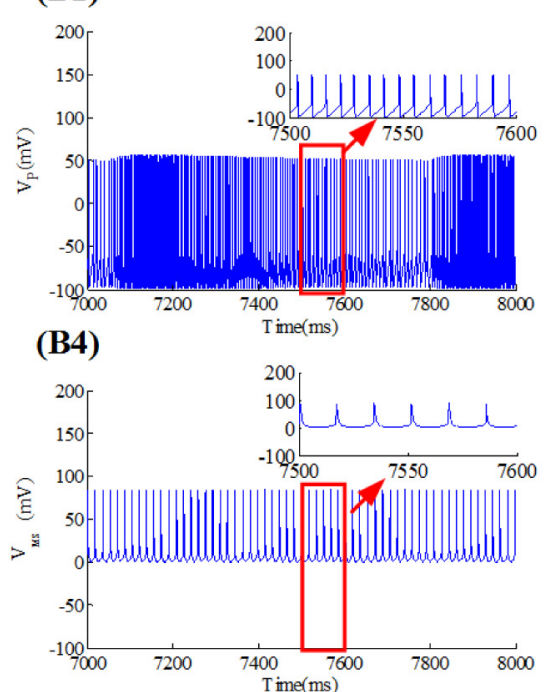

(A2)

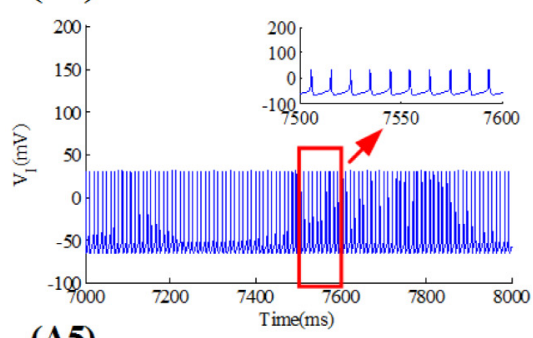

(A5)

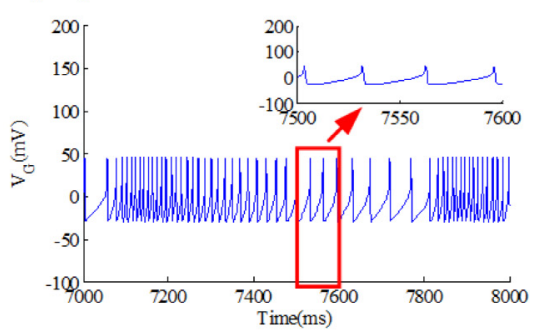

(B2)

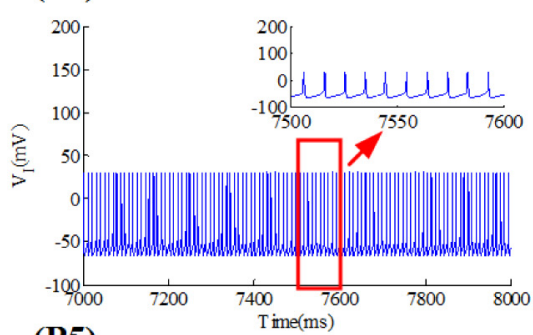

(B5)

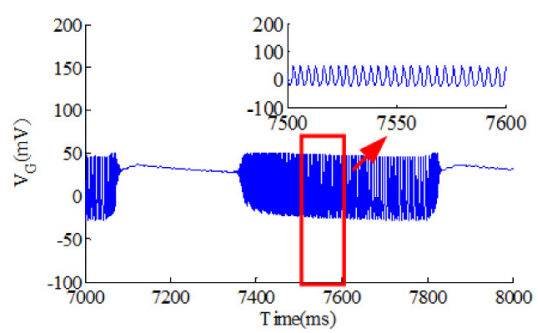

(A3)

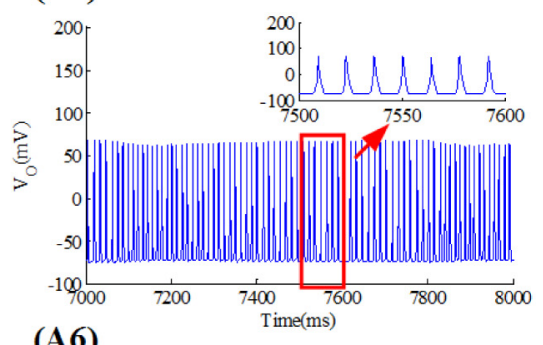

(A6)

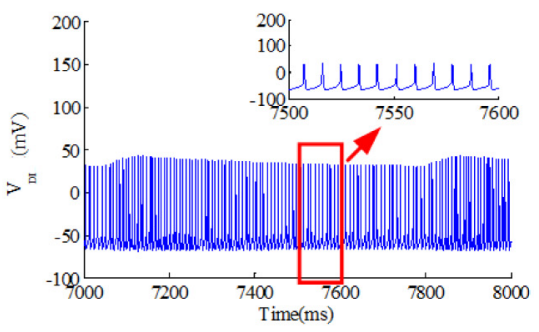

(B3)

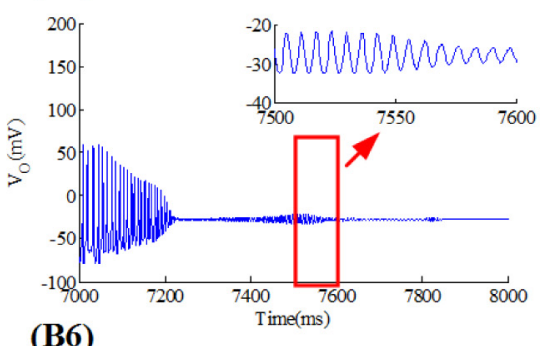

(B6)

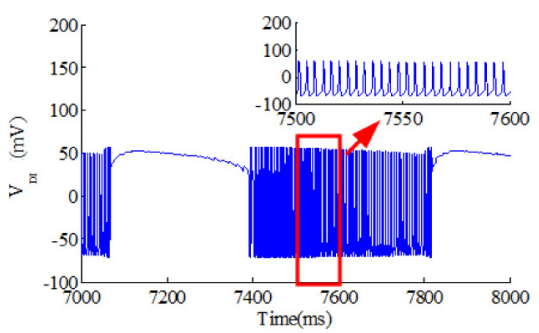

FIG. 2. Time series (of period from $7000 \mathrm{~ms}$ to $8000 \mathrm{~ms}$ ) and detail (from $7500 \mathrm{~ms}$ to $7600 \mathrm{~ms}$ ) corresponding to simultaneous effects of BDNF and neuronal noise on the DG-CA3 network. (a) Potentials obtained with $c_{B D N F}=4 \mu \mathrm{mol}$ and Mean $I=2 \mu \mathrm{A} / \mathrm{cm}^{2}$. (a1): P, (a2): I, (a3): O, (a4): MS, (a5): G, and (a6): DI. (b) $c_{B D N F}=32 \mu \mathrm{mol}$ and Mean $I=2 \mu \mathrm{A} / \mathrm{cm}^{2}$, where (b1): P, (b2): I, (b3): O, (b4): MS, (b5): G, and (b6): DI. (c) $c_{B D N F}=4 \mu \mathrm{mol}$ and $M e a n I=6 \mu \mathrm{A} / \mathrm{cm}^{2}$, where (c1): P, (c2): I, (c3): O, (c4): MS, (c5): G, and (c6): DI. (d) $c_{B D N F}=32 \mu \mathrm{mol}$ and Mean I $=6 \mu \mathrm{A} / \mathrm{cm}^{2}$, where (d1): P, (d2): I, (d3): O, (d4): MS, (d5): G, and (d6): DI. 
(C1)

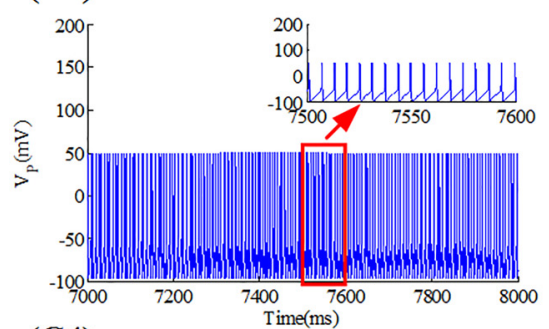

(C4)

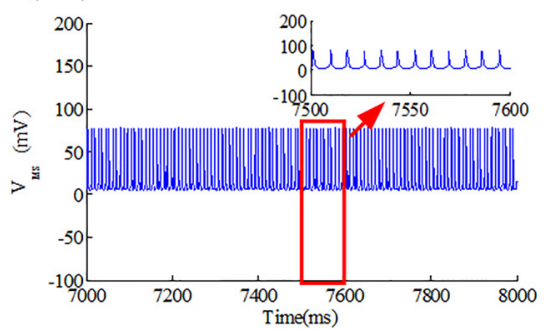

(D1)

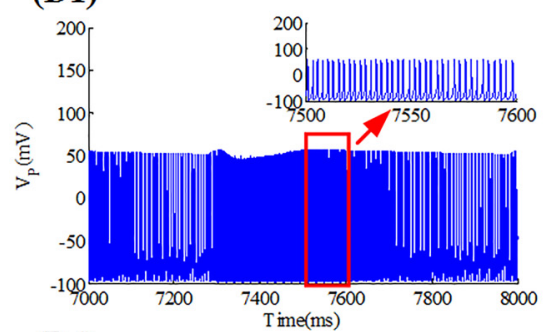

(D4)

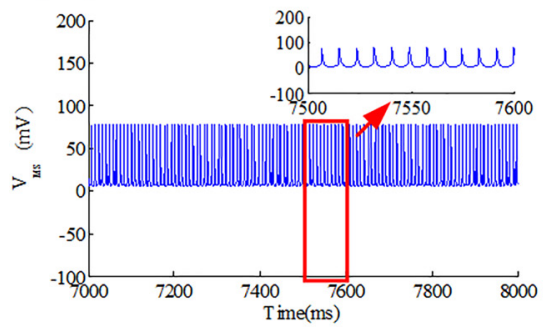

(C2)

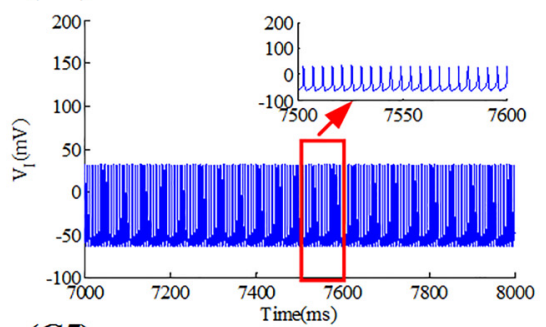

(C5)

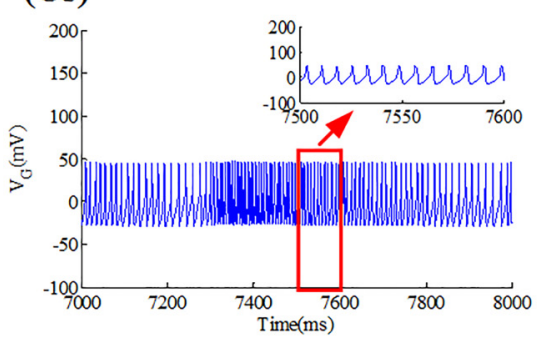

(D2)

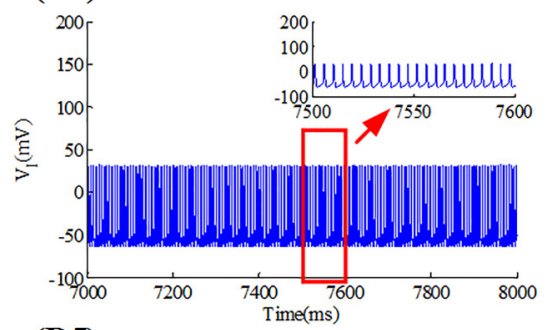

(D5)

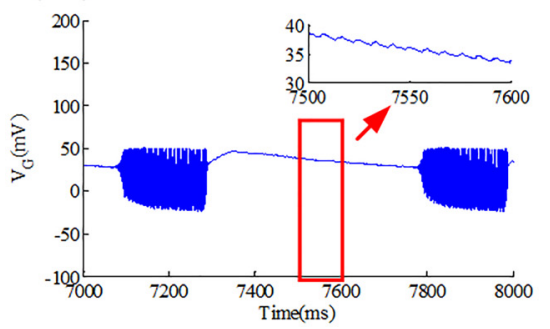

(C3)

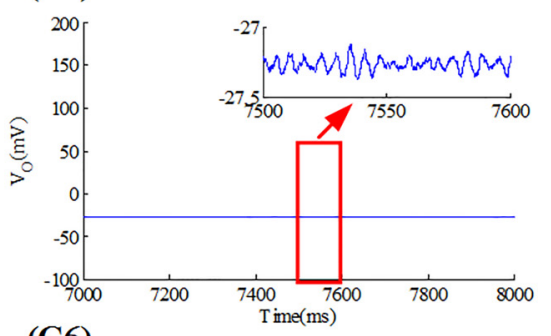

(C6)

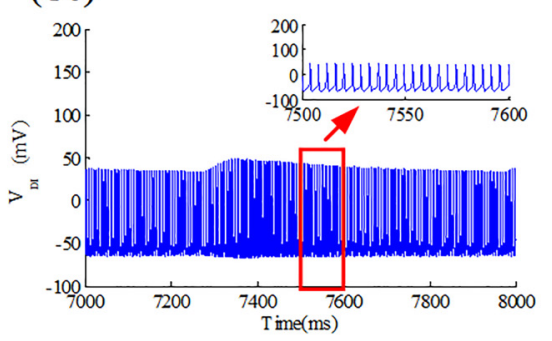

(D3)

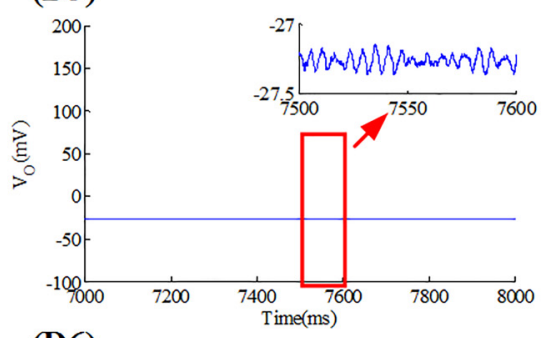

(D6)

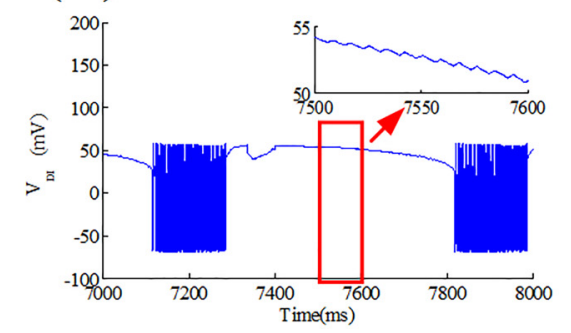

FIG. 2. Continued.

\section{Network synchronization}

To quantify correlations between neurons, Pearson's correlation coefficient ${ }^{56}$ is one of the most common measures. It is defined as the ratio of the covariance of samples $x$ and $y$ and

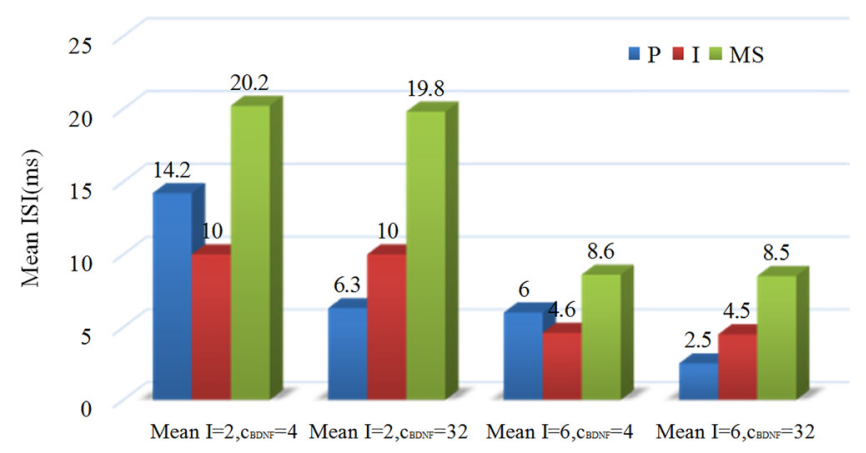

FIG. 3. Bars of mean ISIs. We consider four conditions: $c_{B D N F}=4 \mu \mathrm{mol}$ and Mean $I=2 \mu \mathrm{A} / \mathrm{cm}^{2}, c_{B D N F}=32 \mu \mathrm{mol}$ and Mean $I=2 \mu \mathrm{A} / \mathrm{cm}^{2}, c_{B D N F}=$ $4 \mu \mathrm{mol}$ and Mean $I=6 \mu \mathrm{A} / \mathrm{cm}^{2}$, and $c_{B D N F}=32 \mu \mathrm{mol}$ and Mean $I=$ $6 \mu \mathrm{A} / \mathrm{cm}^{2}$. The blue bars indicate mean ISIs of P, the red bars mean ISIs of I, and the green bars mean ISIs of MS. the product of their respective standard deviations, i.e.,

$$
r=\frac{\operatorname{cov}(x, y)}{\sqrt{\sigma_{x}^{2} \sigma_{y}^{2}}},
$$

where cov means covariance. $\sigma_{x}$ represents the standard deviation of sample $x$, and $\sigma_{y}$ indicates the standard deviation of sample $y . r$ varies from -1 to $+1 .|r|=1$ means perfect correlation between $x$ and $y \cdot|r|=0$ indicates no (zero-lag) correlation between them.

In addition, we focus on the synchronization evolution of the entire network in response to effects exerted by BNDF and neural noise. Synchronization of a network with $N$ oscillators can be quantitatively described by the synchronization factor $\eta$ given as ${ }^{57}$

$$
\begin{gathered}
V(t)=\frac{1}{N} \sum_{i=1}^{N} V_{i}(t), \\
\sigma_{V}^{2}=\left\langle[V(t)]^{2}\right\rangle_{t}-\left[\langle V(t)\rangle_{t}\right]^{2}, \\
\sigma_{V_{i}}^{2}=\left\langle\left[V_{i}(t)\right]^{2}\right\rangle_{t}-\left[\left\langle V_{i}(t)\right\rangle_{t}\right]^{2},
\end{gathered}
$$


(A1)

(B1)

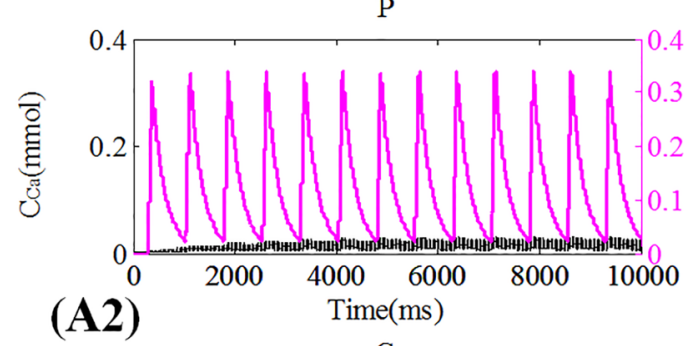

G
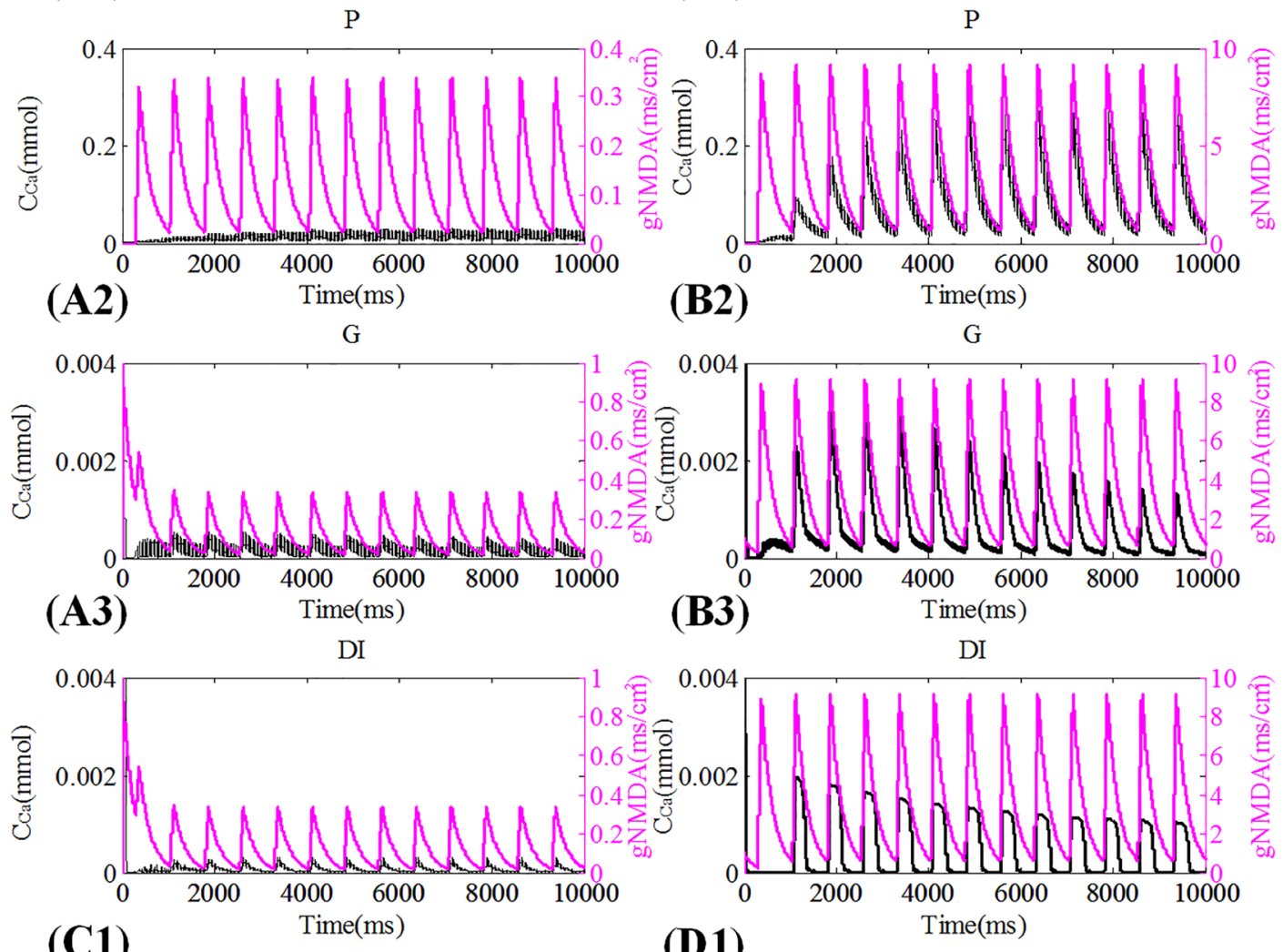

(C1)

$\mathrm{P}$

$P$
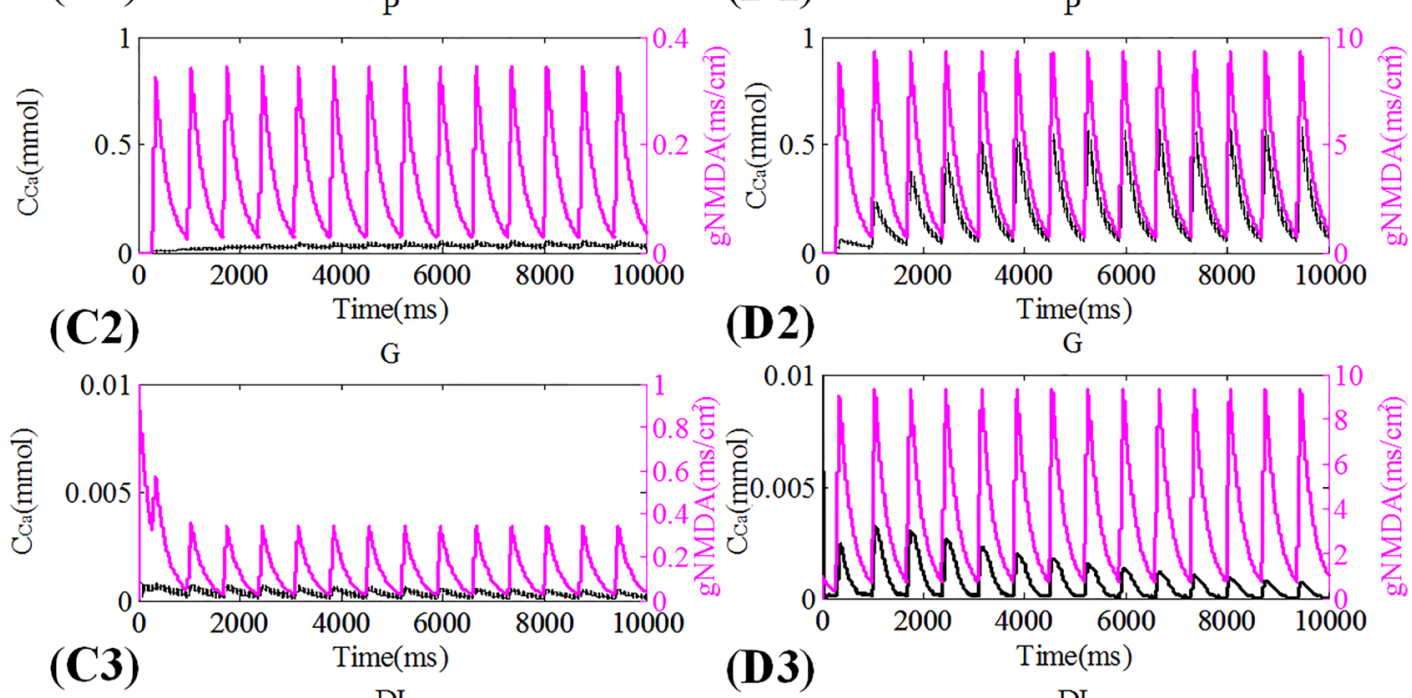

(D2)

G

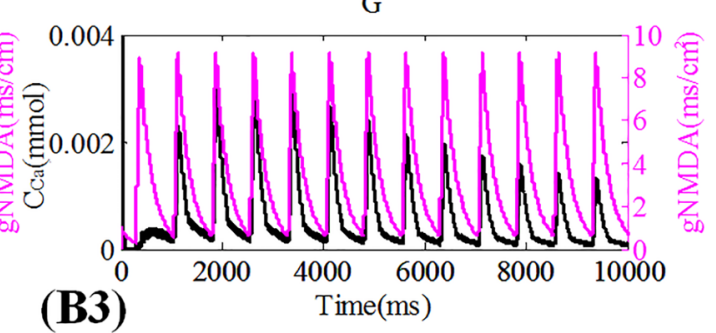

(B3)
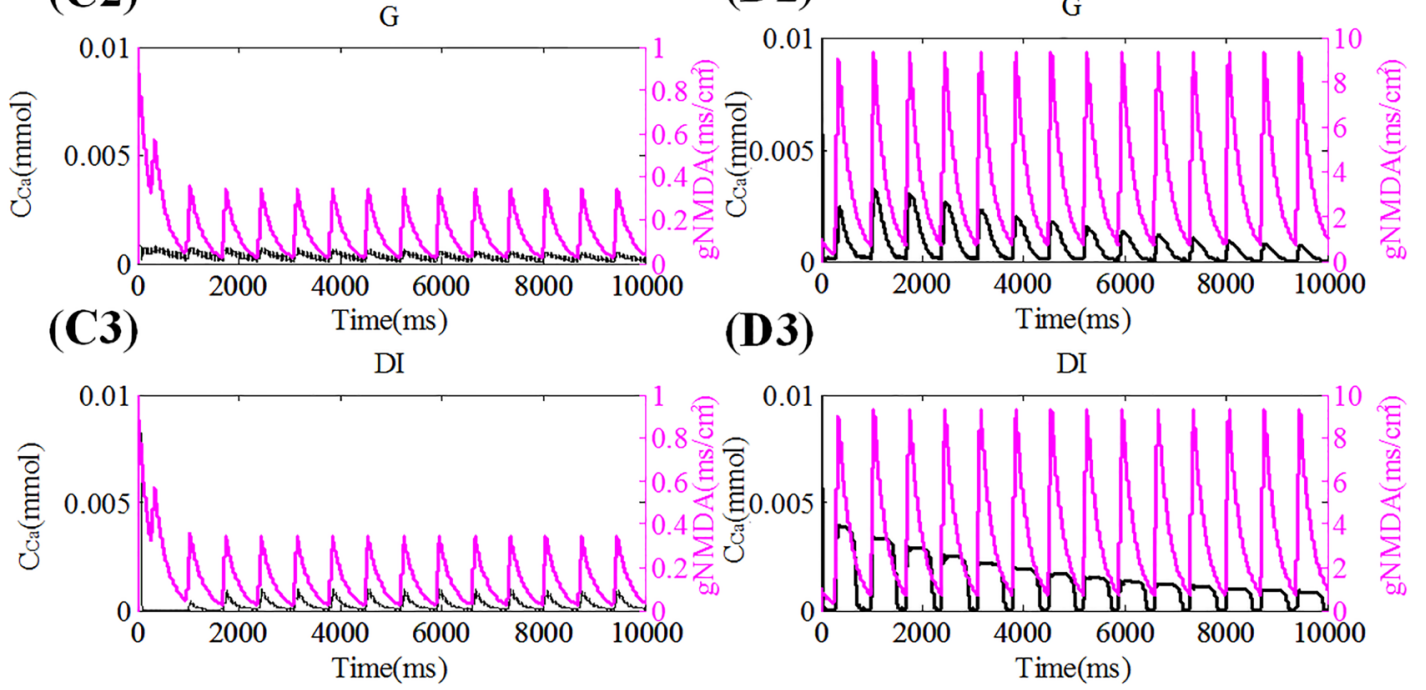

FIG. 4. The temporal evolution of the concentration of $\mathrm{Ca}^{2+}$ and the activation of NMDA receptors, respectively, in typical fast spiking neuron and bursting neurons. Black lines denote the concentration of $\mathrm{Ca}^{2+}$; pink lines are the variations of the NMDA receptors. (a) $c_{B D N F}=4 \mu \mathrm{mol}$ and $M e a n I=2 \mu \mathrm{A} / \mathrm{cm}^{2}$, where (a1) is P, (a2) represents G, and (a3) denotes DI. (b) $c_{B D N F}=32 \mu \mathrm{mol}$ and Mean $I=2 \mu \mathrm{A} / \mathrm{cm}^{2}$, where (b1) shows P, (b2) is G, and (b3) denotes DI. (c) $c_{B D N F}=4 \mu \mathrm{mol}$ and Mean $I=6 \mu \mathrm{A} / \mathrm{cm}^{2}$, where (c1) shows P, (c2) represents G, and (c3) denotes DI. (d) $c_{B D N F}=32 \mu \mathrm{mol}$ and $M e a n I=6 \mu \mathrm{A} / \mathrm{cm}^{2}$, where (d1) is $\mathrm{P},(\mathrm{d} 2)$ represents $\mathrm{G}$, and $(\mathrm{d} 3)$ denotes DI. 


$$
\eta^{2}(N)=\frac{\sigma_{V}^{2}}{\frac{1}{N} \sum_{i=1}^{N} \sigma_{V_{i}}^{2}},
$$

where $V(t)$ is the mean membrane potentials of a neuron. Mean membrane potentials are calculated simultaneously for the six individual membrane potentials. $V_{i}(t)$ indicates the membrane potential of an individual neuron. $\langle\ldots\rangle_{t}=$ $\frac{1}{T_{m}} \int_{0}^{T_{m}} d t$ denotes time-averaging over a time interval $T_{m} . \sigma_{V}$ is the standard deviation of $V(t)$; similarly, $\sigma_{V_{i}}$ is the standard deviation of $V_{i}(t)$. In our network, we employ six types of neurons to construct the DG-CA3 network; therefore, the number of coupled oscillators $N=6 . \eta$ varies from 0 to 1 , where $\eta=0$ means that the network is in a non-synchronized state and $\eta=1$ means that the network is completely synchronized.

\section{SIMULATION RESULTS}

\section{A. Effects of BDNF and noise on individual neurons}

Usually, interictal and preictal spikes are observed before the onset of an epileptic event which is characterized by abnormal oscillations persisting for a few seconds. ${ }^{58}$ Increased amounts of $\mathrm{Mg}^{2+}$ ions are removed by excessive NMDA receptors, and the glutamate can bind with NMDA receptors triggering ictal-like burst discharges. To study the effects of the BDNF concentration pulse and neuronal noise, we compare four situations: (A) low BDNF concentration with low mean neuronal noise, (B) high BDNF concentration with low mean neuronal noise, (C) low BDNF concentration with high mean neuronal noise, and (D) high BDNF concentration with high mean neuronal noise. To acquire a steady signal, the simulation time is set to $10 \mathrm{~s}$, and a (presumably stationary) period of $1 \mathrm{~s}$ from the simulation (from $7000 \mathrm{~ms}$ to $8000 \mathrm{~ms}$ ) is selected. We first consider time series of neuronal potentials in response to the influence exerted by BDNF and neuronal noise on the DG-CA3 network.

It is seen from Fig. 2(a) that for the case when $c_{B D N F}=$ $4 \mu \mathrm{mol}$ and Mean $I=2 \mu \mathrm{A} / \mathrm{cm}^{2}$, all neurons show spiking. Especially, a low BDNF concentration with low noise levels can induce spiking at various frequencies. Next, we increase the concentration of BDNF at a fixed neural noise level, i.e., $c_{B D N F}=32 \mu \mathrm{mol}$ and Mean $I=2 \mu \mathrm{A} / \mathrm{cm}^{2}$. As shown in Fig. 2(b1), neuron $\mathrm{P}$ displays spiking with higher frequency in comparison with the situation in Fig. 2(a1). Similarly, I and MS also demonstrate spiking, and this is shown in Figs. 2(b1) and 2(b4), respectively. However, neuron O performs damped oscillations displayed in Fig. 2(b3). It seems that strong BDNF concentration prevents $\mathrm{O}$ from spiking with high amplitude. In addition, a higher BDNF concentration triggers bursting in both G and DI [shown in Figs. 2(b5) and 2(b6), respectively]. It is worth noting that the whole time series of $\mathrm{G}$ shows one big burst (duration time is about $400 \mathrm{~ms}$ ) and splits into small bursts (duration time is about $200 \mathrm{~ms}$ ). The membrane potentials between bursts are positive, so they do not seem to need the process of repolarization. Likewise, DI shows spiking, followed by a positive membrane potential between bursts. Next, we consider low BDNF concentration with high mean neuronal noise, i.e., $c_{B D N F}=4 \mu \mathrm{mol}$ and Mean $I=6 \mu \mathrm{A} / \mathrm{cm}^{2}$. In comparison with Fig. 2(a), neurons spike at a higher frequency [shown in Fig. 2(c)]. However, the hyper-excitatory neuron I transmits more inhibitory input to $\mathrm{O}$, which inhibits $\mathrm{O}$ in the form of subthreshold oscillation [see details in Fig. 2(c3)]. Lastly, we set both $c_{B D N F}$ and Mean $I$ to the high values, i.e., $c_{B D N F}=32 \mu \mathrm{mol}$ and Mean $I=6 \mu \mathrm{A} / \mathrm{cm}^{2}$. It can be seen from Fig. 2(d) that the enhanced mean value of noise accompanied by the increased concentration of BDNF can induce fast spiking in P, I, and MS (here we define "fast" spiking as having a frequency over $100 \mathrm{~Hz}$ ) and hinder $\mathrm{O}$ from discharging dramatically. We also see the process of splitting of spiking activity into shorter bursts for both $\mathrm{G}$ and DI.

In addition, to test whether the combined effects of BDNF and neuronal noise can promote $\mathrm{P}, \mathrm{I}$, and MS discharging at higher frequencies, we calculate their mean interspike intervals (ISI). Statistic cubes in Fig. 3 provide a visualization of mean ISIs under the four conditions. We can observe that the mean ISI of $\mathrm{P}$ is the smallest value of all neurons. In line with this, the individual and combined effects also reduce the mean ISIs belonging to I and MS, respectively. In summary, the enhanced effects of BDNF and neuronal noise individually and combined can abnormally elevate the spiking frequency of neurons in the DG-CA3 network.

Next, we consider the calcium concentration and the activation of NMDA receptors in typical fast spiking and bursting neurons, respectively. We study four sets of parameters as in Fig. 2. Figure 4 shows the evolution of the concentrations
(A)

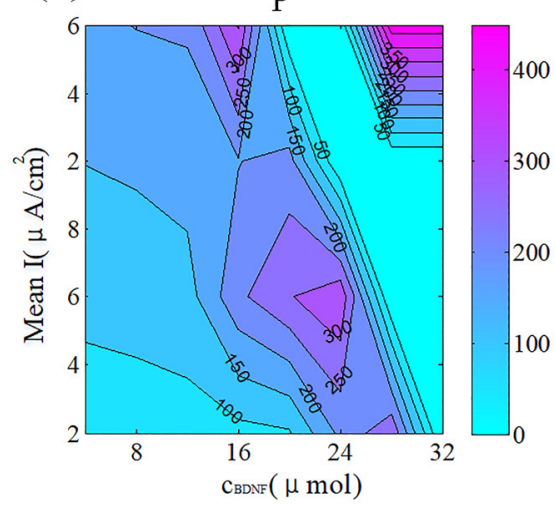

(B)

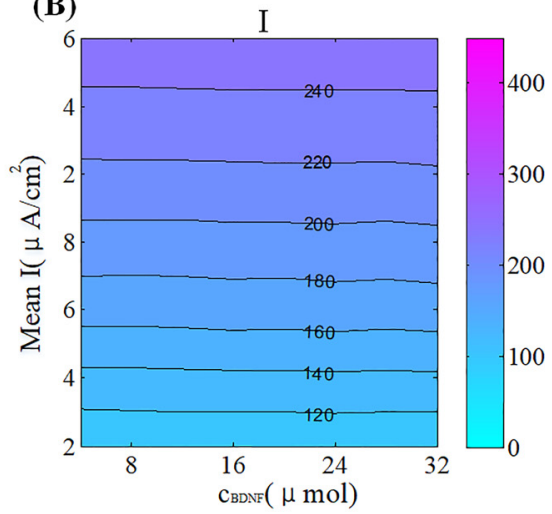

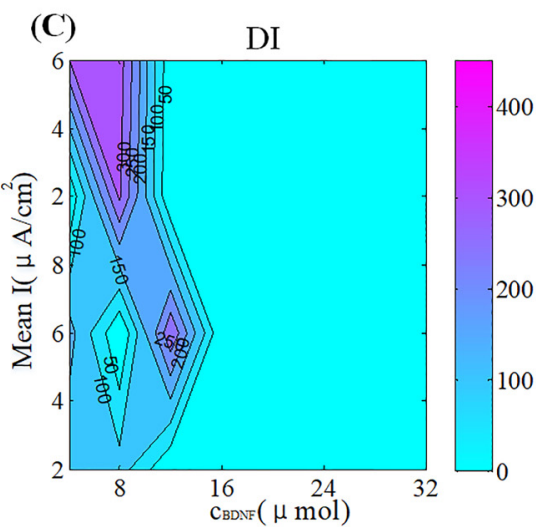

FIG. 5. Contour maps of the dominant neuronal frequency. Different color represents different frequency (see color bar on the right). (a): P, (b): I, and (c): DI. 
of $\mathrm{Ca}^{2+}$ and NMDA receptors under these conditions. In Figs. 4(a1), 4(b1), 4(c1), and 4(d1), we see strong oscillations of the concentration of $\mathrm{Ca}^{2+}$ in post-synaptic neuron $\mathrm{P}$. As the combined effects of BDNF and neuronal noise are gradually enhanced, the oscillation of $\mathrm{Ca}^{2+}$ increase in amplitude and becomes sharper. Also, the transition from low to higher amplitude oscillation of the activation of NMDA receptors can be observed. The overexpression of BDNF together with the enhanced noise foster the activation of NMDA receptors, and this in turn allows $\mathrm{Ca}^{2+}$ to permeate to the pre-synaptic neuron, which activates spiking. Moreover, we can see from Figs. 4(a2) and 4(c2) that the concentration of $\mathrm{Ca}^{2+}$ and the activation of NMDA receptors oscillate with low amplitude when low BNDF and low neuronal noise is applied to G. As the impact becomes stronger, the amplitudes get larger [shown in Figs. 4(b2) and 4(d2)]. Similar results can be found in DI, shown in Figs. 4(a3), 4(b2), 4(c3), and 4(d3), where not only NMDA receptors are activated but also the concentration of $\mathrm{Ca}^{2+}$ is elevated, resulting in ictogenesis. Besides, we can also observe that $\mathrm{P}$ is characterized by the presence of pre-ictal discharges, while $\mathrm{G}$ and DI show burst discharges or ictal-like burst events (compared to $\mathrm{P}$ ).

To investigate the frequency characteristics of neurons under the influence of BDNF and noise, we calculated the dominant frequency in the neurons of DG and CA3, respectively. As shown in Fig. 5(a), P exhibits complex frequency characteristics. In general, the dominant frequency of $\mathrm{P}$ rises when the impact of BDNF and noise is set to moderate values. Nevertheless, the picture is complex with a low frequency region separating a second high frequency region where both BDNF and noise are large. This could present hyper-excited fast spiking. In contrast, I demonstrates the simple frequency characteristics shown in Fig. 5(b), where the dominant frequencies corresponding to I change monotonically as the noise level increases. Here, BDNF has little impact on the dominant frequencies, while these neurons are sensitive to the noise level. In addition, domain frequency panels corresponding to $\mathrm{O}, \mathrm{MS}$, and $\mathrm{G}$ are similar to panel (b), i.e., there is a monotonous increase as a function of parameter in each case. DI shows high frequency oscillations when BDNF is small and noise is strong, as shown in Fig. 5(c). As the concentration of BDNF is increased beyond a value of 16 , the dominant frequency does not change significantly any more, which implies that at this value DI transits to a discharge dynamics of slow frequency bursts. In brief, the effects of BDNF and noise have a great influence on $\mathrm{P}, \mathrm{G}$, and DI, induce a high concentration of $\mathrm{Ca}^{2+}$, lead to irregular fast spiking or ictal-like activity, and result in complex dominant frequency transitions for some parameter combinations.

\section{B. Effects of BDNF and noise on the entire network}

In this section, we will describe the response of the entire network as a function of BDNF and noise. Figure 6 shows the mean membrane potentials at different levels of BDNF and noise. It is observed in Fig. 6(a) that the responses of the network initially drops, which is caused varying BDNF at a fixed noise level. When a high concentration of BDNF is applied to the DG-CA3 network, the amplitude varies from $-20 \mathrm{mv}$ to $0 \mathrm{mv}$ in a sawtooth manner. Consistent with the above approach, we then enhance the strength of the neuronal noise. It is seen from Fig. 6(b) that the potential traces with the value from $-20 \mathrm{mv}$ to $0 \mathrm{mv}$ extend their scope during the injection of higher neuronal noise. Larger sawtooth-like waves evoked by the enhanced noise indicate that the regions of discharges (under $-20 \mathrm{mV}$ ) are compensated by the regions of amplitude (above $-20 \mathrm{mV}$ ). We conclude that the effects of BDNF and noise lead the network potentials to transit from low amplitude discharges to high amplitude discharges. This implies an increase in the excitability of the DG-CA3 network.

In Fig. 7, we show the correlations calculated according to Eq. (2) at Mean $I=2 \mu \mathrm{A} / \mathrm{cm}^{2}, 4 \mu \mathrm{A} / \mathrm{cm}^{2}$, and $8 \mu \mathrm{A} / \mathrm{cm}^{2}$, respectively. Each small square represents the color-coded correlation at fixed noise with varying BDNF (increasing from left to right). There are eight correlation columns (from left to right) in each panel, representing linear changes in BDNF from 4. The majority of correlation coefficients in Fig. 7(a) are positive but small, which indicates that neurons interact with each other weakly. Only G and DI are strongly correlated, which suggests that G and DI mutually promote discharges. In contrast, $\mathrm{P}$ and $\mathrm{O}$ are negatively correlated for small BDNF, which could mean that their discharges are reciprocally inhibited. As noise becomes stronger, i.e., Mean $I=4 \mu \mathrm{A} / \mathrm{cm}^{2}$ [see in Fig. 7(b)], all correlation coefficients become more positive, even though overall they remain small. The correlation between G and DI remains strong. Further increase of noise level does not result in further increase

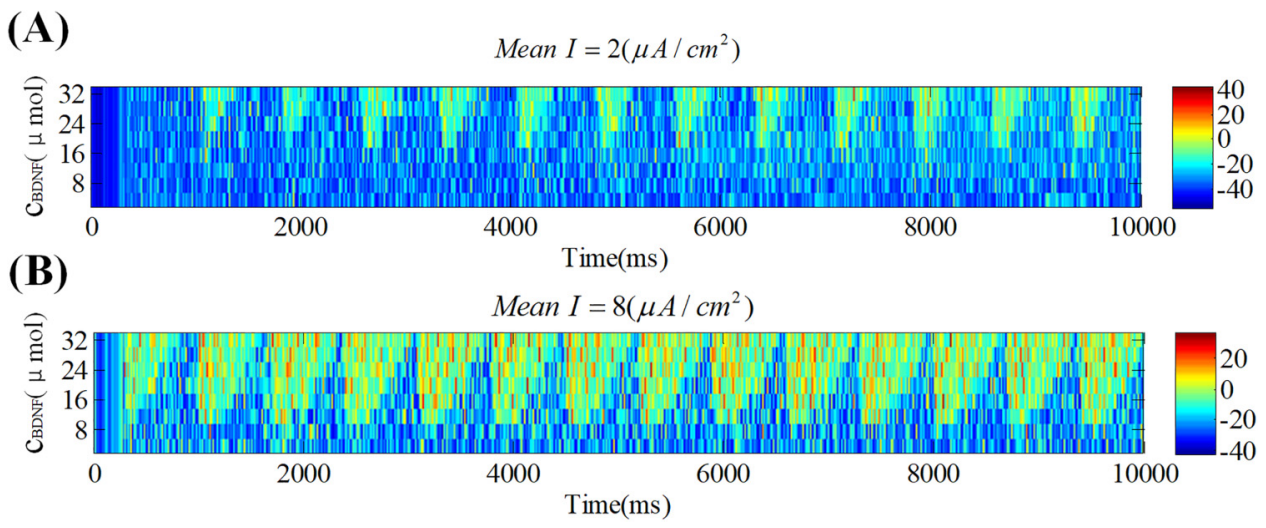

FIG. 6. Transitions of the mean membrane potentials in response to varying levels of BNDF and noise. Different colors express different values of the mean membrane potentials. (a): Mean $I=2 \mu \mathrm{A} / \mathrm{cm}^{2}$ and (b): Mean $I=$ $8 \mu \mathrm{A} / \mathrm{cm}^{2}$. 

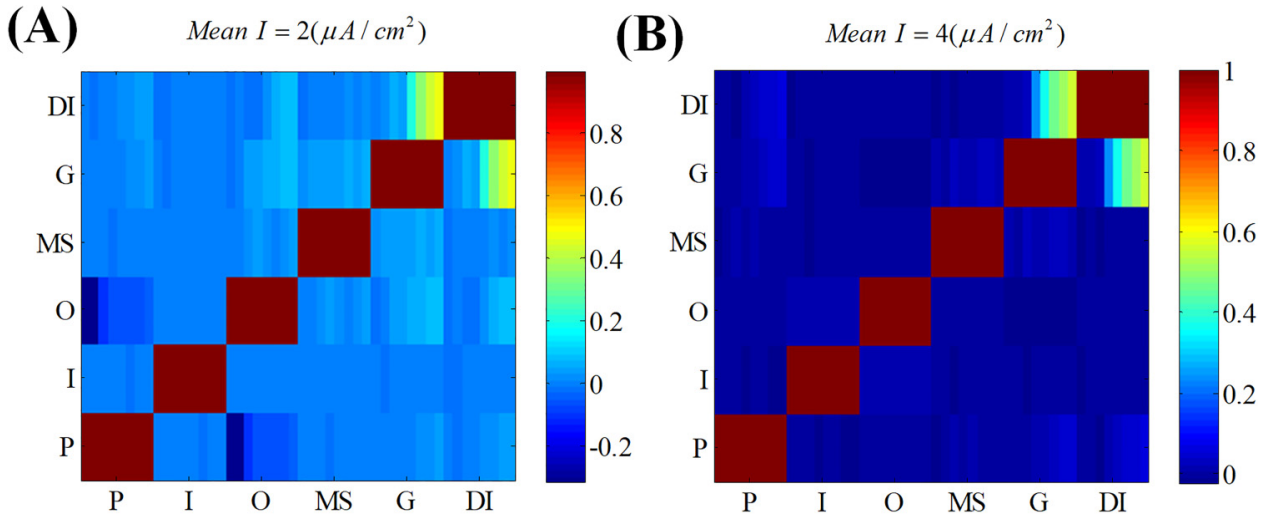

FIG. 7. Correlation matrix for the DG-CA3 network neurons. Different color means different correlation coefficients. (a) Variable BDNF at Mean $I=$ $2 \mu \mathrm{A} / \mathrm{cm}^{2}$ and (b)Mean $I=4 \mu \mathrm{A} / \mathrm{cm}^{2}$. of the correlations. This illustrates that correlations vary most when noise is weak. However, beyond a certain threshold, the correlation does not appear to increase further. Additionally, dramatic changes of correlations between $\mathrm{G}$ and DI from low to high correlation can be seen from the corresponding squares in Figs. 7(a) and 7(b), which indicates that (in contrast to the noise) BDNF plays a significant role in promoting the interaction between $\mathrm{G}$ and DI.

Finally, we investigate synchronization of the DG-CA3 network. Figure 8 shows synchronization curves as a function of BDNF at different levels of mean noise. At small noise level is small, i.e., Mean $I=2 \mu \mathrm{A} / \mathrm{cm}^{2}$; the synchronization of the entire network strongly increases with increasing BDNF. As the noise level increases, the increase in synchronization occurs more slowly. At high noise levels, the synchronization shows a more complex dependence with an increase followed by a decrease for high concentrations of BDNF (i.e., $B D N F=24 \mu \mathrm{mol}$ and Mean $I=8 \mu \mathrm{A} / \mathrm{cm}^{2}$ ), which indicates that overexpressed BDNF together with strong noise suppresses further synchronization of the entire network.

To conclude, the combined effects of BDNF and noise facilitate the transition process from discharge at low potential amplitudes to higher amplitudes by increasing the excitability

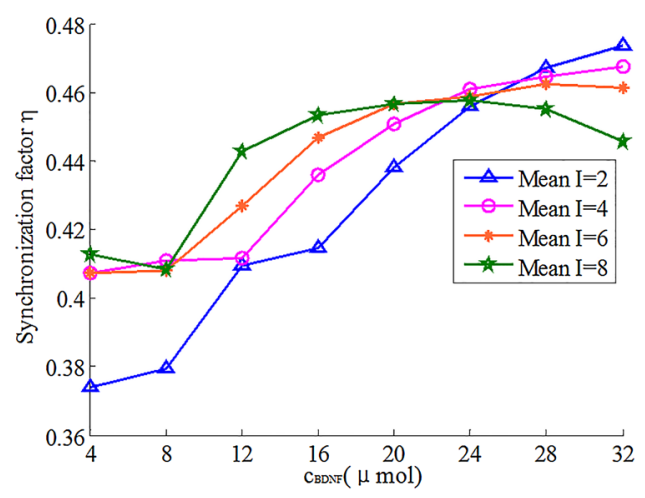

FIG. 8. Synchronization evolution of the entire network with respect to variable BDNF concentrations at fixed mean noise, corresponding to Mean $I=$ $2 \mu \mathrm{A} / \mathrm{cm}^{2}$ (blue line with triangle), $I=4 \mu \mathrm{A} / \mathrm{cm}^{2}$ (magenta line with circle), $I=6 \mu \mathrm{A} / \mathrm{cm}^{2}$ (brown line with hexagram), and $8 \mu \mathrm{A} / \mathrm{cm}^{2}$ (green line with asterisk), respectively. of the network, resulting in increased synchronization of the whole DG-CA3 network.

\section{CONCLUSION}

TLE is characterized by abnormal synchronous neuronal fast spiking or bursting within the hippocampus. To reveal the molecular and the neuronal noise-induced mechanisms behind the transition from normal spiking to abnormal activity during TLE seizures, we studied a biophysically inspired mathematical model of a hippocampal network in regions CA3 and DG. We modified a previously proposed network model by including the effects of BDNF and neuronal noise and systematically investigated the dynamics in the time and frequency domain of individual neurons and the entire network. We show that the increasing BDNF and neuronal noise activate more NMDA receptors owing to the membrane permeability of $\mathrm{Ca}^{2+}$, which in return induces preictal discharges characterized by irregular fast spiking and ictal-like bursting events, respectively. Increased excitation of individual neurons is found, resulting in an increased dominant firing frequency. Furthermore, the investigation at the network level indicates that the effects play a crucial role in enhancing the overall amplitude of the membrane potential. The enhancement strengthens both correlation and synchronization of the hippocampal network. Our results may thus provide an insight into the possible mechanisms leading to abnormal spiking and bursting and thereby help explain the transition to seizure dynamics in TLE. A challenge of future research is to add more units to account for the interplay between the hippocampus and cortex, e.g., region Cornu Ammonis 1 (CA1) and entorhinal cortex (EC), to obtain an integrated map of activity from the cellular neuronal networks to the clinically relevant macroscopic ictogenic network.

\section{ACKNOWLEDGMENTS}

This research was supported by the National Natural Science Foundation of China (Grant Nos. 11325208, 11572015, and 11772019). This paper is to celebrate the 70th birthday of Hans Braun from the Institute of Physiology, University of Marburg, Germany. We sincerely wish Hans good health and all the best! 


\section{APPENDIX A: MODEL OF GRANULE CELL VOLTAGE}

We employ the somatic model proposed by Yuen and Durand $^{49}$ to simulate the voltage of a granule cell:

$$
\begin{aligned}
C \frac{d V_{G}}{d t}= & g_{N a} m^{3} \mathrm{~h}\left(V_{N a}-V_{G}\right)+g_{K} n^{4}\left(V_{K}-V_{G}\right) \\
& +g_{s k} q^{2}\left(V_{K}-V_{G}\right)+\mathrm{g}_{L}\left(V_{L}-V_{G}\right)+I_{C a^{2+}-G} \\
& +I_{G},
\end{aligned}
$$

$$
\frac{d x}{d t}=\alpha_{x}(1-x)-\beta_{x} x \text { for } x=m, h, n, \text { or } q,
$$

$$
\begin{gathered}
\alpha_{m}\left(V_{G}\right)=\frac{-0.3\left(V_{G}-9\right)}{\exp \left[\left(V_{G}-9\right) /(-5)\right]-1}, \\
\beta_{m}\left(V_{G}\right)=\frac{0.3\left(V_{G}-37\right)}{\exp \left[\left(V_{G}-37\right) / 5\right]-1}, \\
\alpha_{h}\left(V_{G}\right)=\frac{0.23}{\exp \left[\left(V_{G}+13\right) / 20\right]}, \\
\beta_{h}\left(V_{G}\right)=\frac{3.33}{\exp \left[\left(V_{G}-39.5\right) /(-10)\right]+1}, \\
\alpha_{n}\left(V_{G}\right)=\frac{-0.007\left(V_{G}-31\right)}{\exp \left[\left(V_{G}-31\right) /(-6)\right]-1}, \\
\beta_{n}\left(V_{G}\right)=\frac{0.264}{\exp \left[\left(V_{G}-6\right) / 40\right]}, \\
\alpha_{q}\left(C a^{2+}\right)=\frac{0.0041}{\exp \left[\left(10 \log C a^{2+}+4.48\right) /(-4.5)\right]}, \\
\operatorname{exp[(10\operatorname {log}Ca^{2+}+36.4)/35]},
\end{gathered}
$$

where $C, I_{G}, V_{G}, t$, and $g$ denote capacitance density, external electrical stimulus (pulse of BDNF or neuronal noise) for the granule cell, membrane potential, time, and conductance density, respectively. The units of the corresponding variables are $\mu \mathrm{F} / \mathrm{cm}^{2}, \mu \mathrm{A} / \mathrm{cm}^{2}, \mathrm{mV}, \mathrm{ms}$, and $\mathrm{ms} / \mathrm{cm}^{2}$. Parameters: $C=3.4, g_{N a}=250, g_{K}=40, g_{L}=0.025, g_{s k}=4.7, V_{N a}=$ $45, V_{K}=-85$, and $V_{L}=-67.46$.

\section{APPENDIX B: MODEL OF MOSSY CELL}

According to Pinsky and Rinzel's model formulation, ${ }^{50}$ the voltage-gated ion channels are described by the following set of differential equations:

$$
\begin{aligned}
C \frac{d V_{M S}}{d t}= & g_{N a} m_{\infty}^{2} h\left(V_{N a}-V_{M S}\right)+g_{K} n\left(V_{K}-V_{M S}\right) \\
& +\frac{g_{c}}{p}\left(V_{M D}-V_{M S}\right)+g_{L}\left(V_{L}-V_{M S}\right)+\frac{I_{M S}}{p},(\mathrm{~B} 1) \\
C \frac{d V_{M D}}{d t}= & g_{C a} s^{2}\left(V_{C a}-V_{M D}\right)+g_{K A P H} q\left(V_{K}-V_{M D}\right) \\
& +g_{K C} \chi(C a)\left(V_{K}-V_{M D}\right)+\frac{g_{c}}{1-p}\left(V_{M S}-V_{M D}\right) \\
& +g_{L}\left(V_{L}-V_{M D}\right)+\frac{I_{M D}}{1-p}+\frac{I_{N M D A}+I_{A M P A}}{p-1},
\end{aligned}
$$

$$
\begin{gathered}
\frac{d \mathrm{Ca}^{2+}}{d t}=0.13 g_{K C} \chi(\mathrm{Ca})\left(V_{K}-V_{M D}\right)-0.0075 \mathrm{Ca}, \\
\frac{d y}{d t}=\frac{y_{\infty}\left(V_{M S, M D}\right)-y}{\tau_{y}\left(V_{M S, M D}\right)} \quad \text { for } y=h, n, s, c, \text { or } q, \\
y_{\infty}\left(V_{M S, M D}\right)=\frac{\alpha_{y}\left(V_{M S, M D}\right)}{\alpha_{y}\left(V_{M S, M D}\right)+\beta_{y}\left(V_{M S, M D}\right)}, \\
\tau_{y}\left(V_{M S, M D}\right)=\frac{1}{\alpha_{y}\left(V_{M S, M D}\right)+\beta_{y}\left(V_{M S, M D}\right)},
\end{gathered}
$$

$$
I_{N M D A}=\frac{g_{N M D A} S_{i}(t)}{1+0.28 \exp \left[-0.062\left(V_{M D}-60\right)\right]}\left(V_{M D}-V_{s y n}\right),
$$

$$
I_{A M P A}=g_{A M P A} W_{i}(t)\left(V_{M D}-V_{s y n}\right),
$$$$
\frac{d S_{i}}{d t}=\sum_{j} H\left(V_{G / D I / P}-10\right)-\frac{S_{i}}{150},
$$

$$
\frac{d W_{i}}{d t}=\sum_{j} H\left(V_{G / P}-10\right)-\frac{W_{i}}{2},
$$$$
H(x)= \begin{cases}1 & x \geq 0 \\ 0 & x<0\end{cases}
$$

$$
\alpha_{m}\left(V_{M S}\right)=\frac{0.32\left(13.1-V_{M S}\right)}{\exp \left[\left(13.1-V_{M S}\right) / 4\right]-1},
$$

$$
\beta_{m}\left(V_{M S}\right)=\frac{0.28\left(V_{M S}-40.1\right)}{\exp \left[\left(V_{M S}-40.1\right) / 5\right]-1},
$$$$
\alpha_{n}\left(V_{M S}\right)=\frac{0.016\left(35.1-V_{M S}\right)}{\exp \left[\left(35.1-V_{M S}\right) / 5\right]-1},
$$$$
\beta_{n}\left(V_{M S}\right)=0.25 \exp \left(0.5-0.025 V_{M S}\right) \text {, }
$$$$
\alpha_{h}\left(V_{M S}\right)=0.128 \exp \left(\left(17-V_{M S}\right) / 18\right),
$$$$
\beta_{h}\left(V_{M S}\right)=\frac{4}{1+\exp \left[\left(40-V_{M S}\right) / 5\right]},
$$

$$
\begin{aligned}
\alpha_{s}\left(V_{M D}\right) & =\frac{1.6}{1+\exp \left[-0.072\left(V_{M D}-65\right)\right]}, \\
\beta_{s}\left(V_{M D}\right) & =\frac{0.02\left(V_{M D}-51.1\right)}{\exp \left[\left(V_{M D}-51.1\right) / 5\right]-1},
\end{aligned}
$$




$$
\begin{aligned}
& \alpha_{c}\left(V_{M D}\right)=\frac{\exp \left[\left(V_{M D}-10\right) / 11\right]-\exp \left[\left(V_{M D}-6.5\right) / 27\right]}{18.975} \\
& \text { for } V_{M D} \leq 50, \\
& \alpha_{c}\left(V_{M D}\right)=2 \exp \left[\left(6.5-V_{M D}\right) / 27\right] \quad \text { for } V_{M D}>50, \quad(\mathrm{~B} 21) \\
& \beta_{c}\left(V_{M D}\right)=2 \exp \left[\left(6.5-V_{M D}\right) / 27\right]-\alpha_{c} \text { for } V_{M D} \leq 50,
\end{aligned}
$$$$
\beta_{c}\left(V_{M D}\right)=0 \text { for } V_{M D}>50
$$

$$
\begin{gathered}
\alpha_{q}\left(V_{M D}\right)=\min \left[(0.00002) C a^{2+}, 0.01\right], \\
\beta_{q}\left(V_{M D}\right)=0.001,
\end{gathered}
$$

with $C=3, g_{N a}=250, g_{K D R}=15, g_{L}=0.1, g_{K A P H}=0.8$, $g_{C a}=10, g_{K C}=15$, and $g_{c}=2.1$. The reversal potentials are $V_{N a}=120, V_{K}=-85, V_{C a}=140, V_{\text {syn }}=60$, and $V_{L}=0$. In addition, $\chi\left(C a^{2+}\right)=\min \left(\mathrm{Ca}^{2+} / 250,1\right) . p=0.5$, which represents the percentage of total area in the soma-like compartment.

\section{APPENDIX C: MODEL OF CA3 AND DG GABAERGIC INTERNEURONS}

The mathematical model of inhibitory interneuron is based on Wang and Buzsáki. ${ }^{48}$ Kinetic equations are given as follows:

$$
\begin{aligned}
& C \frac{d V_{I, D I}}{d t}=g_{N a} m_{\infty}\left(V_{I, D I}\right)^{3} h\left(V_{N a}-V_{I, D I}\right) \\
& +g_{K} n^{4}\left(V_{K}-V_{I, D I}\right)+g_{L}\left(V_{L}-V_{I, D I}\right) \\
& +I_{C a^{2+}-I, D I}+I_{I, D I}, \\
& \frac{d C a^{2+}}{d t}=-\frac{C a^{2+}}{\tau_{d}}-\frac{I_{C a^{2+}-I}}{w z F}, \\
& I_{C a^{2+-I}}=g_{c a} e^{2} w\left(V_{C a}-V_{I}\right), \\
& \frac{d x}{d t}=\frac{x_{\infty}\left(V_{I, D I}\right)-x}{\tau_{x}\left(V_{I, D I}\right)} \quad \text { for } x=m, w, e, h, \text { or } n, \\
& x_{\infty}\left(V_{I, D I}\right)=\frac{\alpha_{x}\left(V_{I, D I}\right)}{\alpha_{x}\left(V_{I, D I}\right)+\beta_{x}\left(V_{I, D I}\right)} \quad \text { for } x=m, w, e, h \text {, or } n, \\
& \tau_{x}\left(V_{I, D I}\right)=\frac{0.2}{\alpha_{x}\left(V_{I, D I}\right)+\beta_{x}\left(V_{I, D I}\right)} \quad \text { for } x=w, e, h, \text { or } n, \\
& \alpha_{m}\left(V_{I, D I}\right)=\frac{0.1\left(V_{I, D I}+35\right)}{1-\exp \left[-\left(V_{I, D I}+35\right) / 10\right]}, \\
& \beta_{m}\left(V_{I, D I}\right)=4 \exp \left[-\left(V_{I, D I}+60\right) / 18\right], \\
& \alpha_{w}\left(V_{I}\right)=\frac{3 \times 10^{-6}}{\exp \left[\left(V_{I}-11.5\right) / 17.5\right]}, \\
& \beta_{w}\left(V_{I}\right)=\frac{3 \times 10^{-4}}{\exp \left[\left(V_{I}-19.5\right) /(-14)\right]+1}, \\
& \alpha_{e}\left(V_{I}\right)=\frac{-0.031\left(V_{I}-24.5\right)}{\exp \left[\left(V_{I}-24.5\right) /(-3)\right]-1},
\end{aligned}
$$

$$
\begin{gathered}
\beta_{e}\left(V_{I}\right)=\frac{0.031\left(V_{I}-40\right)}{\exp \left[\left(V_{I}-40\right) / 3\right]-1}, \\
\alpha_{h}\left(V_{I, D I}\right)=0.07 \exp \left[-\left(V_{I, D I}+58\right) / 20\right], \\
\beta_{h}\left(V_{I, D I}\right)=\frac{1}{\exp \left[-0.1\left(V_{I, D I}+28\right)\right]+1}, \\
\alpha_{n}\left(V_{I, D I}\right)=\frac{0.01\left(V_{I, D I}+34\right)}{1-\exp \left(-0.1\left(V_{I, D I}+34\right)\right)}, \\
\beta_{n}\left(V_{I, D I}\right)=0.125 \exp \left[-\left(V_{I, D I}+44\right) / 80\right],
\end{gathered}
$$

where $C=1, V_{N a}=55, V_{K}=-90, V_{L}=-65, V_{C a}=70$, $g_{N a}=35, g_{K}=9, g_{C a}=1$, and $g_{L}=0.1$.

\section{APPENDIX D: MODEL OF PYRAMIDAL CELL}

The pyramidal cell is simulated with the following set of equations: ${ }^{51}$

$$
\begin{aligned}
& C \frac{d V_{p}}{d t}=g_{N a} m_{\infty}\left(V_{p}\right)^{3} h\left(V_{N a}-V_{p}\right)+g_{K} n^{4}\left(V_{K}-V_{p}\right) \\
& +g_{L}\left(V_{L}-V_{p}\right)+I_{C a^{2+}-P}+I_{p}, \\
& \frac{d x}{d t}=\frac{x_{\infty}\left(V_{P}\right)-x}{\tau_{x}\left(V_{P}\right)} \quad \text { for } x=m, h, \text { or } n, \\
& x_{\infty}\left(V_{P}\right)=\frac{\alpha_{x}\left(V_{P}\right)}{\alpha_{x}\left(V_{P}\right)+\beta_{x}\left(V_{P}\right)} \quad \text { for } x=m, h \text {, or } n, \\
& \tau_{x}\left(V_{P}\right)=\frac{1}{\alpha_{x}\left(V_{P}\right)+\beta_{x}\left(V_{P}\right)} \quad \text { for } x=h \text { or } n \\
& \alpha_{m}\left(V_{P}\right)=\frac{0.32\left(V_{P}+54\right)}{1-\exp \left[-\left(V_{P}+54\right) / 4\right]}, \\
& \beta_{m}\left(V_{P}\right)=\frac{0.28\left(V_{P}+27\right)}{\exp \left[\left(V_{P}+27\right) / 5\right]-1}, \\
& \beta_{h}\left(V_{P}\right)=\frac{4}{1+\exp \left[-\left(V_{P}+27\right) / 5\right]}, \\
& \alpha_{n}\left(V_{P}\right)=\frac{0.032\left(V_{P}+52\right)}{1-\exp \left[-\left(V_{P}+52\right) / 5\right]}, \\
& \beta_{n}\left(V_{P}\right)=0.5 \exp \left[-\left(V_{P}+57\right) / 40\right]
\end{aligned}
$$

The following set of parameters is used: $C=1, V_{N a}=50$, $V_{K}=-100, V_{L}=-67, g_{N a}=100, g_{K}=80$, and $g_{L}=0.1$. 


\section{APPENDIX E: MODEL OF O-LM CELL}

O-LM cell is modeled by Tort's et al.'s equations, ${ }^{52}$ which are as follows:

$$
\begin{aligned}
& C \frac{d V_{O}}{d t}=g_{N a} m^{3} \mathrm{~h}\left(V_{N a}-V_{O}\right)+g_{K} n^{4}\left(V_{K}-V_{O}\right) \\
& +g_{A} \mathrm{ab}\left(V_{A}-V_{O}\right)+g_{h} r\left(V_{h}-V_{O}\right) \\
& +\mathrm{g}_{L}\left(V_{L}-V_{O}\right)+I_{C a^{2+}-O}+I_{O}, \\
& \frac{d C a^{2+}}{d t}=-\frac{C a^{2+}}{\tau_{d}}-\frac{I_{C a^{2+}-I}}{w z F}, \\
& I_{C a^{2+}-O}=g_{c a} e^{2} w\left(V_{C a}-V_{I}\right), \\
& \frac{d x}{d t}=\frac{x_{\infty}\left(V_{O}\right)-x}{\tau_{x}\left(V_{O}\right)} \quad \text { for } x=m, w, e, h, n, a, b \text {, or } r, \\
& x_{\infty}\left(V_{O}\right)=\frac{\alpha_{x}\left(V_{O}\right)}{\alpha_{x}\left(V_{O}\right)+\beta_{x}\left(V_{O}\right)} \quad \text { for } x=m, w, e, h \text {, or } n, \\
& \tau_{x}\left(V_{O}\right)=\frac{1}{\alpha_{x}\left(V_{O}\right)+\beta_{x}\left(V_{O}\right)} \quad \text { for } x=m, w, e, h \text {, or } n, \\
& \alpha_{m}\left(V_{O}\right)=\frac{-0.1\left(V_{O}+38\right)}{\exp \left[-\left(V_{O}+38\right) / 10\right]-1}, \\
& \alpha_{w}\left(V_{O}\right)=\frac{3 \times 10^{-6}}{\exp \left[\left(V_{O}-11.5\right) / 17.5\right]}, \\
& \beta_{w}\left(V_{O}\right)=\frac{3 \times 10^{-4}}{\exp \left[\left(V_{O}-19.5\right) /(-14)\right]+1}, \\
& \alpha_{e}\left(V_{O}\right)=\frac{-0.031\left(V_{O}-24.5\right)}{\exp \left[\left(V_{O}-24.5\right) /(-3)\right]-1}, \\
& \beta_{e}\left(V_{O}\right)=\frac{0.031\left(V_{O}-40\right)}{\exp \left[\left(V_{O}-40\right) / 3\right]-1}, \\
& \beta_{m}\left(V_{O}\right)=4 \exp \left[-\left(V_{O}+65\right) / 18\right], \\
& \alpha_{h}\left(V_{O}\right)=0.07 \exp \left[-\left(V_{O}+63\right) / 20\right], \\
& \beta_{h}\left(V_{O}\right)=\frac{1}{1+\exp \left[-\left(V_{O}+33\right) / 10\right]}, \\
& \alpha_{n}\left(V_{O}\right)=\frac{0.018\left(V_{O}-25\right)}{1-\exp \left[-\left(V_{O}-25\right) / 25\right]}, \\
& \beta_{n}\left(V_{O}\right)=\frac{0.0036\left(V_{O}-35\right)}{\exp \left[\left(V_{O}-35\right) / 12\right]-1}, \\
& a_{\infty}\left(V_{O}\right)=\frac{1}{1+\exp \left[-\left(V_{O}+14\right) / 16.6\right]}, \\
& \tau_{a}\left(V_{O}\right)=5, \\
& b_{\infty}\left(V_{O}\right)=\frac{1}{1+\exp \left[\left(V_{O}+71\right) / 7.3\right]}, \\
& \tau_{b}\left(V_{O}\right)=\frac{1}{\frac{0.000009}{\left.\exp \left(V_{O}-26\right) / 18.5\right]}+\frac{0.014}{0.2+\exp \left[-\left(V_{O}+70\right) / 11\right]}},
\end{aligned}
$$

$$
r_{\infty}\left(V_{O}\right)=\frac{1}{1+\exp \left[\left(V_{O}+84\right) / 10.2\right]},
$$

$$
\tau_{r}\left(V_{O}\right)=\frac{1}{\exp \left(-14.59-0.086 V_{O}\right)+\exp \left(-1.87+0.0701 V_{O}\right)},
$$

where $C=1.3, V_{K}=-100, V_{A}=-90, V_{h}=-32.9, V_{C a}=70$, $V_{L}=-70, V_{N a}=90, g_{N a}=30, g_{K}=23, g_{C a}=1, g_{L}=0.05$, $g_{A}=16$, and $g_{h}=12$.

\section{APPENDIX F: RECEPTORS AND CALCIUM MODELS MEDIATED BY BDNF}

Dynamical equation of NMDA receptors relying on BDNF can be modeled as ${ }^{47,59}$

$$
\begin{aligned}
\frac{d g_{N M D A-x}}{d t}= & k_{b N}+k_{g N} \frac{C_{B D N F}}{K_{g N}+C_{B D N F}}-k_{d N} g_{N M D A-x}, \\
& \text { for } x=G, D I, \text { and } P,
\end{aligned}
$$

where $k_{b N}=5 \times 10^{-8} \mathrm{mS} / \mathrm{cm}^{2} \mathrm{~ms}^{-1}, \quad k_{g N}=1 \times 10^{-7} \mathrm{mS} /$ $\mathrm{cm}^{2} \mathrm{~ms}^{-1}, k_{d N}=0.004 \mathrm{~ms}^{-1}$, and $K_{g N}=50 \mu \mathrm{M}$.

Dynamic equation of AMPA receptors determined by $\mathrm{Ca}^{2+}$ can be described as ${ }^{47,59}$

$$
\frac{d g_{A M P A-x}}{d t}=k_{b A}+k_{g A} \frac{C a^{2+}{ }_{x}}{K_{g A}+C a^{2+}{ }_{x}}-k_{d A} g_{A M P A-x},
$$

for $x$ is suitable for $G, M S, P, I$, and $O$,

where $\quad k_{b A}=1 \times 10^{-7} \mathrm{mS} / \mathrm{cm}^{2} \mathrm{~ms}^{-1}, \quad k_{g A}=5 \times 10^{-6} \mathrm{mS} /$ $\mathrm{cm}^{2} \mathrm{~ms}^{-1}, k_{d A}=0.002 \mathrm{~ms}^{-1}$ and $K_{g A}=0.2 \mu \mathrm{M}$.

In terms of $\mathrm{Ca}^{2+}$, equation depending on activation of NMDA receptors is given as ${ }^{47,59}$

$\frac{d C a^{2+} x}{d t}=k_{b}+k_{N} g_{N M D A-x} S_{x}-\frac{C a^{2+} x}{\tau_{C a}}, \quad$ for $x=G, D I$, and $P$,

where $k_{b}=1 \times 10^{-4} \mathrm{mMms}^{-1}, \quad k_{N}=0.02 \mathrm{mM} \mathrm{ms}^{-1}$, and $\tau_{C a}=9 \mathrm{~ms} . S_{x}$ is the weight of NMDA receptors, whose form is similar to Eq. (B9). Thus, $I_{\mathrm{Ca}^{2+}{ }_{x}}$ has its form ${ }^{49}$

$$
I_{C a^{2+}-x}=g_{c a-x} s_{x}^{2}\left(V_{C a-x}-V_{x}\right), \quad \text { for } x=G, D I \text {, and } P,
$$

where $g_{C a-G}=g_{C a-P}=g_{C a-D I}=1 \mathrm{mS} / \mathrm{cm}^{2}$ and $V_{C a-G}=$ $V_{C a-P}=V_{C a-D I}=70 \mathrm{mV}$.

\section{APPENDIX G: MODEL OF SYNAPSE}

We employ Ermentrout's and Kopell's ${ }^{53}$ synaptic model to imitate the dynamics of neuronal connectivity. The synaptic gating variable $s$ is defined as $0 \leq s \leq 1$ and obeys the rule:

$$
\frac{d s}{d t}=\rho(V) \frac{1-s}{\tau_{R}}-\frac{s}{\tau_{D}},
$$

where $V$ is the membrane potential and $\rho$ represents the Heaviside function

$$
\rho(V)=\frac{1+\tanh (V / 4)}{2},
$$

where $\tau_{R}$ and $\tau_{D}$ are the rise and decay time constants, respectively. The mathematical expression of the synaptic model is 
given as

$$
I_{s y n}=g_{i j} s_{i}(t)\left(V_{r e v}-V_{j}\right),
$$

where $V_{r e v}$ is the synaptic reversal potential, $g_{i j}$ denotes the conductance strength, and $s_{i}$ denotes the gating variable. Furthermore, $V_{j}$ is the membrane potential of neuron $j$.

$G$ receives an AMPA type synaptic current and an NMDA type synaptic current from $\mathrm{MS}$, and receives a $\mathrm{GABA}_{\mathrm{A}}$ type synaptic current from DI.

$$
\begin{aligned}
I_{s y n G}= & I_{s y n \_M S-G}+I_{s y n \_D I-G} \\
= & I_{A M P A \_M S-G}+I_{N M D A \_M S-G}+I_{G A B A_{A} \_D I-G}, \\
= & g_{A M P A-G} S_{M S}(t)\left(V_{r e v \_A M P A}-V_{G}\right) \\
& +\frac{g_{N M D A-G} S_{M S-G}(t)}{1+0.28 \exp \left[-0.062\left(V_{G}-60\right)\right]}\left(V_{G}-V_{s y n_{-} G}\right) \\
& +g_{D I-G} S_{D I}(t)\left(V_{r e v \_G A B A_{A}}-V_{G}\right),
\end{aligned}
$$

where $\quad g_{D I-G}=0.001, \quad V_{s y n_{-} G}=60, \quad V_{\text {rev_AMPA }}=0, \quad$ and $V_{\text {rev_GABA }}=-80$.

MD receives an AMPA type synaptic current from $G$ and $\mathrm{P}$, respectively, and a $\mathrm{GABA}_{\mathrm{A}}$ type synaptic current from DI.

$$
\begin{aligned}
& I_{\text {syn } M D}=I_{\text {syn_G-MD }}+I_{\text {syn_P-MD }}+I_{\text {syn_DI-G }} \\
& =I_{A M P A_{-} G-M D}+I_{A M P A_{-} P-M D}+I_{G A B A_{A} D I-M D} \text {, } \\
& =g_{A M P A \_G-D I} W_{i}(t)\left(V_{M D}-V_{s y n \_M D}\right) \\
& +g_{A M P A-M D} s_{P}(t)\left(V_{\text {rev_AMPA }}-V_{M D}\right) \\
& +g_{D I-M D} s_{D I}(t)\left(V_{r e v \_G A B A_{A}}-V_{M D}\right),
\end{aligned}
$$

where $g_{D I-M D}=0.001$ and $V_{\text {syn_MD }}=60$.

DI receives an AMPA type synaptic current and an NMDA type synaptic current from MS and receives an AMPA type synaptic current from $\mathrm{G}$.

$$
\begin{aligned}
I_{\text {synDI }}= & I_{\text {syn } \_M S-D I}+I_{\text {syn_G-DI }} \\
= & I_{A M P A \_M S-D I}+I_{N M D A \_M S-D I}+I_{A M P D \_D-D I}, \\
= & g_{A M P A-D I} S_{M S}(t)\left(V_{\text {rev_AMPA }}-V_{D I}\right) \\
& +\frac{g_{N M D A-D I} S_{M S-D I}(t)}{1+0.28 \exp \left[-0.062\left(V_{D I}-60\right)\right]}\left(V_{D I}-V_{s y n \_D I}\right) \\
& +g_{A M P A-D I} S_{D I}(t)\left(V_{r e v \_A M P A}-V_{D I}\right),
\end{aligned}
$$

where $V_{\text {syn_DI }}=60$.

$P$ receives an AMPA type synaptic current and an NMDA type synaptic current from MS. It also receives a $\mathrm{GABA}_{\mathrm{A}}$ type synaptic current from I and O, respectively.

$$
\begin{aligned}
& I_{s y n G}=I_{\text {syn_MS-P }}+I_{\text {syn_I-P }}+I_{\text {syn_O-P }} \\
& =I_{A M P A \_M S-P}+I_{N M D A \_M S-P}+I_{G A B A_{A} \_} I-P \\
& +I_{G A B A_{A-} O-P} \text {, } \\
& =g_{A M P A-P} s_{M S}(t)\left(V_{\text {rev_AMPA }}-V_{P}\right) \\
& +\frac{g_{N M D A-P} S_{M S-P}(t)}{1+0.28 \exp \left[-0.062\left(V_{P}-60\right)\right]}\left(V_{P}-V_{s y n_{-} P}\right) \\
& +g_{I-P} S_{I}(t)\left(V_{\text {rev_GABA }}-V_{P}\right) \\
& +g_{O-P} s_{O}(t)\left(V_{\text {rev_GABA }}-V_{P}\right) \text {, }
\end{aligned}
$$

where $g_{I-P}=0.001, g_{O-P}=0.001$, and $V_{s y n_{-} P}=60$.
I receives an AMPA type synaptic current from $\mathrm{P}$ and receives a $\mathrm{GABA}_{\mathrm{A}}$ type synaptic current from $\mathrm{O}$.

$$
\begin{aligned}
I_{\text {synI }}= & I_{\text {syn_P-I}}+I_{\text {syn_O-I }}=I_{A M P A \_P-I}+I_{G A B A_{A} \_-I}, \\
= & g_{A M P A-I} S_{P}(t)\left(V_{\text {rev_AMPA }}-V_{I}\right) \\
& +g_{O-I} S_{O}(t)\left(V_{\text {rev_GABA }}-V_{I}\right),
\end{aligned}
$$

where $g_{O-I}=0.001$.

Finally, $\mathrm{O}$ receives an AMPA type synaptic current from $\mathrm{P}$ and $\mathrm{GABA}_{\mathrm{A}}$ type synaptic current from I.

$$
\begin{aligned}
I_{\text {synO } O}= & I_{\text {syn } \_P-O}+I_{\text {syn } \_} I-O=I_{A M P A \_P-O}+I_{G A B A_{A} \_} I-O, \\
= & g_{A M P A-O} S_{P}(t)\left(V_{\text {rev_AMPA }}-V_{O}\right) \\
& +g_{I-O} S_{I}(t)\left(V_{\text {rev_GABA }}-V_{O}\right),
\end{aligned}
$$

where $g_{I-O}=0.001$.

${ }^{1}$ P. De Guzman, Y. Inaba, G. Biagini et al., "Subiculum network excitability is increased in a rodent model of temporal lobe epilepsy," Hippocampus 16, 843-860 (2006).

${ }^{2}$ V. Cutsuridis, S. Cobb, and B. P. Graham, "Encoding and retrieval in a model of the hippocampal CA1 microcircuit," Hippocampus 20, 423-446 (2010).

${ }^{3}$ A. W. L. Chiu, S. S. Jahromi, H. Khosravani et al., "The effects of high-frequency oscillations in hippocampal electrical activities on the classification of epileptiform events using artificial neural networks," J. Neural Eng. 3, 9-20 (2006).

${ }^{4}$ S. Eftekhari, S. Mehrabi, M. Soleimani et al., "BDNF modifies hippocampal KCC2 and NKCC1 expression in a temporal lobe epilepsy model," Acta Neurobiol. Exp. 74, 276-287 (2014).

${ }^{5}$ H. E. Scharfman, Brain-Derived Neurotrophic Factor (BDNF) and the Dentate Gyrus Mossy Fibers: Implications for Epilepsy (Springer, 2005), pp. 201-220.

${ }^{6}$ Y. Akira and C. P. Martha, "Post-synaptic BDNF-TrkB signaling in synapse maturation, plasticity and disease," Dev. Neurobiol. 70(5), 304-322 (2010).

${ }^{7}$ V. Chiglieri, C. Sgobio, S. Patassini et al., "TrkB/BDNF-dependent striatal plasticity and behavior in a genetic model of epilepsy: Modulation by valproic acid," Neuropsychopharmacology 35, 1531-1540 (2010).

${ }^{8}$ H. E. Scharfman, "Brain-derived neurotrophic factor and epilepsy-A missing link?," Epilepsy Curr. 5(3), 83-88 (2005).

${ }^{9}$ H. M. S. Shaari, B. S. Haerian, L. Baum et al., "Association of BDNF polymorphisms with the risk of epilepsy: A multicenter study," Mol. Neurobiol. 53(5), 2869-2877 (2016).

${ }^{10}$ H. E. Scharfman, J. H. Goodman, and A. L. Sollas, "Actions of brainderived neurotrophic factor in slices from rats with spontaneous seizures and mossy fiber sprouting in the dentate gyrus," J. Neurosci. 19(13), 5619-5631 (1999).

${ }^{11}$ R. Koyama and Y. Ikegaya, "To BDNF or not to BNNF: That is the epileptic hippocampus," Neuroscientist 11(4), 282-287 (2005).

${ }^{12}$ J. O. McNamara and H. E. Scharfman, "Temporal lobe epilepsy and the BDNF receptor, TrkB," Epilepsia 51(Suppl. 5), 46 (2010).

${ }^{13}$ X. P. He, R. Koyloski, S. Nef et al., "Conditional deletion of TrkB but not BDNF prevents epileptogenesis in the kindling model," Neuron $\mathbf{4 3}(1)$, 31-42 (2004).

${ }^{14}$ S. M. Garraway, J. C. Petruska, and L. M. Mendell, "BDNF sensitizes the response of lamina II neurons to high threshold primary afferent inputs," Eur. J. Neurosci. 18, 2467-2476 (2003).

${ }^{15}$ D. Langemann, L. Pellerin, and A. Peters, "Making sense of AMPA receptor trafficking by modeling molecular mechanisms of synaptic plasticity," Brain Res. 1207, 60-72 (2008).

${ }^{16}$ C. Luscher and R. C. Malenka, "NMDA receptor-dependent long-term potentiation and long-term depression (LTP/LTD)," Cold Spring Harb. Perpect. Biol. 4, a005710 (2012).

${ }^{17}$ R. J. Delorenze, D. A. Sun, and L. S. Deshpande, "Cellular mechanisms underlying acquired epilepsy: The calcium hypothesis of the induction and maintenance of epilepsy," Pharmacol. Ther. 105(3), 229-266 (2005).

${ }^{18}$ E. R. G. Sanabria, H. Su, and Y. Yaari, "Initiation of network bursts by $\mathrm{Ca}^{2+}$-dependent intrinsic bursting in the rat pilocarpine model of temporal lobe epilepsy," J. Physiol. 531(1), 205-216 (2001). 
${ }^{19}$ D. A. Sun, S. Sombati, R. E. Blair et al., "Long-lasting alterations in neuronal calcium homeostasis in an in vitro model of stroke-induced epilepsy," Cell Calcium 35, 155-163 (2004).

${ }^{20} \mathrm{G}$. W. Zamponi, P. Lory, and E. P. Reyes, "Role of voltage-gated calcium channels in epilepsy," Pfluger Arch. 460(2), 395-403 (2010).

${ }^{21}$ A. Neishabouri and A. A. Faisal, "Axonal noise as a source of synaptic variability,” PLoS Comput. Biol. 10(5), e1003615 (2014).

${ }^{22}$ F. Moss, L. M. Ward, and W. G. Sannita, "Stochastic resonance and sensory information processing: A tutorial and review of application," Clin. Neurophysiol. 115, 267-281 (2004).

${ }^{23}$ S. Tanabe and K. Pakdaman, "Noise-enhanced neuronal reliability," Phys. Rev. E 64(4), 091904.

${ }^{24}$ M. W. Slutzky, P. Cvitanovic, and D. J. Mogul, "Deterministic chaos and noise in three in vitro hippocampal models of epilepsy," Ann. Biomed. Eng. 29, 607-618 (2001).

${ }^{25}$ B. Lindner and L. Schinmansky-Geier, "Maximizing spiking train coherence or incoherence in the leaky integrate-and-fire model," Phys. Rev. E 66, 031916 (2002)

${ }^{26}$ M. Jedynak, A. J. Pons, J. G. Ojalvo et al., "Temporally correlated fluctuations drive epileptiform dynamics," Neuroimage 146, 188-196 (2017).

${ }^{27}$ Q. Lu, H. Gu, Z. Yang et al., "Dynamics of firing patterns, synchronizations and resonances in neuronal electrical activities: Experiments and analysis,' Acta Mech. Sin. 24, 593-628 (2008).

${ }^{28}$ X. Sun, J. Lei, M. Perc et al., "Effects of channel noise on firing coherence of small-world Hodgkin-Huxley neuronal networks," Eur. Phys. J. B 79, 61-66 (2011)

${ }^{29}$ X. Sun, M. Perc, Q. Lu et al., "Effects of correlated Gaussian noise on the mean firing rate and correlations of an electrically coupled neuronal network," Chaos 20, 033116 (2010).

${ }^{30} \mathrm{~W}$. Zhang and P. S. Buckmaster, "Dysfunction of the dentate basket cell circuit in a rat model of temporal lobe epilepsy," J. Neurosci. 29(4), 7846-7856 (2009).

${ }^{31}$ S. Postnova, C. Finke, W. Jin et al., "A computational study of the interdependencies between neuronal impulse pattern, noise effects and synchronization,” J. Physiol. Paris 104(3-4), 176-189 (2010).

${ }^{32}$ D. Guo, C. Xia, S. Wu et al., "Stochastic fluctuations of permittivity coupling regulate seizure dynamics in partial epilepsy," Sci. China Technol. Sci. 60(7), 995-1002 (2017).

${ }^{33}$ D. Fan, Q. Wang, and M. Perc, "Disinhibition-induced transitions between absence and tonic-clonic epileptic seizures," Sci. Rep. 5, 12618 (2015).

${ }^{34}$ A. Proddutur, J. Yu, F. Elgammal et al., "Seizure-induced alterations in fast-spiking basket cell GABA currents modulate frequency and coherence of gamma oscillation in network simulations," Chaos 23(4), 046109 (2013).

${ }^{35}$ Z. P. Kilpartrick and P. C. Bressloff, "Effect of synaptic depression and adaptation on spatiotemporal dynamics of an excitatory neuronal network," Phys. D 239(9), 547-560 (2010).

${ }^{36}$ M. Amiri, F. Bahrami, M. Janahmadi et al., "Functional contributions of astrocytes in synchronization of a neuronal network model," J. Theor. Biol. 292(1), 60-70 (2012).

${ }^{37}$ D. Fan, S. Liu, and Q. Wang, "Stimulus-induced epileptic spike-wave discharges in thalamocortical model with disinhibition," Sci. Rep. 6, 37703 (2016).

${ }^{38}$ R. D. Traub and R. K. Wong, "Cellular mechanism of neuronal synchronization in epilepsy," Science 216(4547), 745-747 (1982).

${ }^{39}$ W. C. Stacey and D. M. Durand, "Noise and coupling affect signal detection and bursting in a simulated physiological neural network," J. Neurophysiol. 88(5), 2598-2611 (2002).
${ }^{40}$ V. Volman, M. Perc, and M. Bazhenov, "Gap junctions and epileptic seizures-Two sides of the same coin?," PLoS One 6, e20572 (2011).

${ }^{41}$ D. Guo, M. Chen, M. Perc et al., "Firing regulation of fast-spiking interneurons by autaptic inhibition," Europhys. Lett. 114, 30001 (2016)

${ }^{42} \mathrm{D}$. Guo, S. Wu, M. Chen et al., "Regulation of irregular neuronal firing by autaptic transmission," Sci. Rep. 6, 26096 (2016).

${ }^{43}$ Q. Wang, M. Perc, Z. Duan et al., "Delay-enhanced coherence of spiral waves in noisy Hodgkin-Huxley neuronal networks," Phys. Lett. A 372(35), 5681-5687 (2008).

${ }^{44}$ Q. Wang, M. Perc, Z. Duan et al., "Synchronization transitions on scalefree neuronal networks due to finite information transmission delays," Phys. Rev. E 80, 026206 (2009)

${ }^{45}$ L. Zhang, D. Fan, and Q. Wang, "Transition dynamics of a dentate gyrusCA3 neuronal network during temporal lobe epilepsy," Front. Comput. Neurosci. 11, 61 (2017).

${ }^{46}$ L. Zhang, D. Fan, and Q. Wang, "Synchronous high-frequency oscillations in inhibitory-dominant network motifs consisting of three dentate gyrusCA3 systems," Chaos 28, 063101 (2018).

${ }^{47}$ A. Desttexhe, Z. F. Mainen, and T. J. Sejnowski, "Kinetic models of synaptic transmission," in Methods in Neuronal Modeling from Ions to Networks (The MIT Press, 1998), Vol. 2, pp. 1-26.

${ }^{48}$ X. J. Wang and G. Buzsáki, "Gamma oscillation by synaptic inhibition in a hippocampal interneuronal network model," J. Neurosci. 16(20), 6042-6413

${ }^{49}$ G. L. Yuen and D. Durand, "Reconstruction of hippocampal granule cell electrophysiology by computer simulation," Neuroscience 41(2-3), 411-423 (1991).

${ }^{50}$ P. F. Pinsky and J. Rinzel, "Intrinsic and network rhythmogenesis in a reduced Traub model for CA3 neurons," J. Comput. Neurosci. 1(1-2), 39-60 (1994).

${ }^{51}$ M. S. Olufsen, M. A. Whittington, M. Camper et al., "New functions for the gamma rhythm: Population tuning and preprocessing for the beta rhythm," J. Comput. Neurosci. 14(1), 33-54 (2003).

${ }^{52}$ A. B. Tort, M. A. Kramer, C. Thom et al., "Dynamic cross-frequency couplings of local field potential oscillations in rat striatum and hippocampus during performance of a T-maze task," Proc. Natl. Acad. Sci. U.S.A. 105(51), 20517-20522 (2008).

${ }^{53}$ G. B. Ermentrout and N. Kopell, "Fine structure of neural spiking and synchronization in the presence of conduction delay," Proc. Natl. Acad. Sci. U.S.A. 95(3), 1259-1264 (1998).

${ }^{54}$ S. Vaynman, Z. Ying, and F. Gomezpinlla, "Hippocampal BDNF mediates the efficacy of exercise on synaptic plasticity and cognition," Eur. J. Neurosci. 20, 2580-2590 (2004).

${ }^{55}$ J. Touboul, G. Hermann, and O. Faugeras, "Noise-induced behaviors in neural mean field dynamics," SIAM J. Appl. Dyn. Syst. 11(1), 49-81 (2011).

${ }^{56}$ L. Egghe and L. Leydesdorff, "The relation between Pearson's correlation coefficient r, and Salton's cosine measure,” J. Am. Soc. Inf. Sci. Technol. 60(5), 1027-1036 (2014).

${ }^{57}$ X. Li, D. Cui, P. Jiruska et al., "Synchronization measurement of multiple neuronal populations," J. Neurophysiol. 98(6), 3341-3348 (2007).

${ }^{58}$ F. Wendling, F. Bartolomei, F. Mina et al., "Interictal spikes, fast ripples and seizures in partial epilepsies-combining multi-level computational models with experimental data," Eur. J. Neurosci. 36, 2164-2177.

${ }^{59}$ C. Bernard, Y. C. Ge, E. Stockley et al., "Synaptic integration of NMDA and non-NMDA receptors in large neuronal network models solved by means of differential equations," Biol. Cybern. 70, 267-273 (1994) 